\title{
Quasi-Toeplitz matrix arithmetic: a MATLAB toolbox*
}

\author{
Dario A. Bini ${ }^{\dagger} \quad$ Stefano Massei $i^{\ddagger} \quad$ Leonardo Robol ${ }^{\S}$
}

June 14, 2018

\begin{abstract}
A Quasi Toeplitz (QT) matrix is a semi-infinite matrix of the kind $A=T(a)+E$ where $T(a)=\left(a_{j-i}\right)_{i, j \in \mathbb{Z}^{+}}, E=\left(e_{i, j}\right)_{i, j \in \mathbb{Z}^{+}}$is compact and the norms $\|a\|_{\mathcal{W}}=\sum_{i \in \mathbb{Z}}\left|a_{i}\right|$ and $\|E\|_{2}$ are finite. These properties allow to approximate any QT-matrix, within any given precision, by means of a finite number of parameters.

QT-matrices, equipped with the norm $\|A\|_{\mathcal{Q} \mathcal{T}}=\alpha\|a\|_{\mathcal{W}}+\|E\|_{2}$, for $\alpha=(1+\sqrt{5}) / 2$, are a Banach algebra with the standard arithmetic operations. We provide an algorithmic description of these operations on the finite parametrization of QT-matrices, and we develop a MATLAB toolbox implementing them in a transparent way. The toolbox is then extended to perform arithmetic operations on matrices of finite size that have a Toeplitz plus low-rank structure. This enables the development of algorithms for Toeplitz and quasi-Toeplitz matrices whose cost does not necessarily increase with the dimension of the problem.

Some examples of applications to computing matrix functions and to solving matrix equations are presented, and confirm the effectiveness of the approach.
\end{abstract}

\section{Introduction}

Toeplitz matrices, i.e., matrices having constant entries along their diagonals, are found in diverse settings of applied mathematics, ranging from imaging to Markov chains, and from finance to the solution of PDEs. These matrices can be of large size, and often they are infinite or semi-infinite in the original mathematical model.

As shown in [17, semi-infinite Toeplitz matrices do not form an algebra; in particular, neither product nor inverses of semi-infinite Toeplitz matrices are still Toeplitz structured in general. However, this property continues to hold up to a compact operator from $\ell^{2}$ onto itself, where $\ell^{2}$ is the linear space formed by sequences $x=\left(x_{i}\right)_{i>0}$ such that $\|x\|_{2}:=\left(\sum_{i=1}^{+\infty}\left|x_{i}\right|^{2}\right)^{1 / 2}<+\infty$. More precisely, the set of semi-infinite Toeplitz matrices plus a compact $\ell^{2}$ operator is a Banach algebra, that is, a Banach space with the $\ell^{2}$ operator norm, closed under matrix multiplication, where the associated operator norm is sub-multiplicative. We refer to such matrices as Quasi-Toeplitz matrices, in short QT matrices. Their computational properties have been investigated in $[2,7,8,13]$.

\footnotetext{
${ }^{*}$ This work has been supported by the GNCS/INdAM project 2018 "Tecniche innovative per problemi di algebra lineare". The authors are members of the research group GNCS.

${ }^{\dagger}$ Dipartimento di Matematica, Pisa, Italy, bini@dm.unipi.it

${ }^{\ddagger} \mathrm{EPF}$ Lausanne, Switzerland, stefano.massei@epfl.ch

$\S$ Institute of Information Science and Technologies "A. Faedo", CNR, Pisa, Italy, leonardo.robol@isti.cnr.it
} 
We provide a description of finitely representable QT matrices, together with the analysis of the computational properties of their arithmetic, moreover we provide an implementation of QT matrices in the form of a MATLAB toolbox called cqt-toolbox (fully compatible with GNU/Octave), where the acronym cqt stands for "Computing with Quasi Toeplitz matrices", and show some examples of applications. The toolbox can be downloaded from https://github.com/numpi/cqt-toolbox

\section{$1.1 \quad$ Motivation}

Matrices of infinite size are encountered in several applications which describe the behavior of systems with a countable number of states, and more generally whenever infinite dimensional objects are involved. Typical examples come from queuing models where the number of states of the stochastic process is infinitely countable, say, it can be represented by the set $\mathbb{Z}$ of relative integers or by the set $\mathbb{Z}^{+}$of positive integers, so that the probability transition matrix is bi-infinite or semiinfinite, respectively. In other models, like the random walk in the quarter plane [31, 24], in the QBD processes [27, and in the more general MG1 and GM1 queues [32, the set of states is discrete and bidimensional, i.e., defined by integer pairs $(i, j)$ where at least one component ranges in an infinite set. Sometimes, these pairs belong to $\mathbb{Z} \times \mathbb{Z}^{+}$or to $\mathbb{Z}^{+} \times \mathbb{Z}^{+}$. In these cases, the probability transition matrix has a block structure with infinitely many blocks and with blocks which have infinite size.

A typical feature shared by many models is that — sufficiently far form the border - the transitions from a state to another depend on their relative positions and are independent of the single state, see for instance the tandem Jackson queue 22] or the random walk in the quarter plane analyzed in 24,31. In these situations, the transition probability matrix is block Toeplitz almost everywhere and its blocks are Toeplitz except for some elements in the upper left corner. In particular, the blocks can be written in the form $T(a)+E$, where $T(a)=\left(a_{j-i}\right)$ is the Toeplitz matrix associated with the sequence $a=\left\{a_{i}\right\}_{i \in \mathbb{Z}}$, while $E$ is a matrix having only a finite number of nonzero entries containing the information concerning the boundary conditions. The computation of interesting quantities related to these models, e.g., the steady state vector, requires to solve quadratic matrix equations whose coefficients are given by the blocks of the transition probability matrix.

The numerical treatment of problems involving infinite matrices is usually performed by truncating the size to a finite large value, by solving the finite problem obtained this way and using this finite solution to approximate part of the solution of the infinite problem.

In 30 , the author analyzes this approach - called the finite section method - for infinite linear systems, providing conditions that ensure the solution of the truncated system to converge to the solution of the infinite one, as the size of the section tends to $+\infty$. The analogous strategy can be adopted for solving matrix equations or computing matrix functions, but - in general - there is no guarantee of convergence. In fact, in 1,26, 28, bad effects of truncation are highlighted when solving infinite quadratic matrix equations arising in the Markov chains framework. In [23] a method is designed for a subclass of bidimensional random walks where the solution can be represented in a special form. In particular, the authors point out the difficulty to apply the matrix geometric method of Marcel Neuts [32, and therefore of solving a quadratic matrix equation, due to the infinite size of the matrix coefficients and of the solution.

Recently, a different approach has been introduced by studying structures that allow finitely represented approximations of infinite matrices and that are preserved by matrix operations. Working with this kind of structured matrices does not require to truncate to finite size in order to carry 
out computations.

In $[2,7,8,13$, the class $\mathcal{Q T}$ of semi-infinite Quasi-Toeplitz (QT) matrices has been introduced. This set is formed by matrices of the kind $A=T(a)+E$ where, in general, $a(z)=\sum_{i \in \mathbb{Z}} a_{i} z^{i}$ is a Laurent series such that $\|a\|_{\mathcal{W}}=\sum_{i=-\infty}^{+\infty}\left|a_{i}\right|$ is finite, and $E$ is a compact correction. Each element of this class can be approximated - at any arbitrary precision - with the sum of a banded Toeplitz $T(\widetilde{a})$ plus a matrix $\widetilde{E}$ with finite support. QT-matrices form a Banach algebra and enable the implementation of an approximate matrix arithmetic which operates on the elements of the class. Using this tool, one can deal with certain classical linear algebra issues just plugging the new arithmetic into the procedures designed for matrices of finite size.

Another intriguing aspect of QT-arithmetic is that it can be easily adapted to finite matrices of the form Toeplitz plus low-rank. This paves the way for efficiently computing functions of Toeplitz matrices, which has been recently raised some attention. See [19] for applications concerning signal processing. In [25,29] computing the matrix exponential of large Toeplitz matrices is required for option pricing with the Merton model.

\subsection{New contributions}

In this paper, by continuing the work started in $[2,7,8,13$, we analyze the representation of QT matrices by means of a finite number of parameters, in a sort of analogy with the finite floating point representation of real numbers. Moreover, we investigate some computational issues related to the definition and the implementation of a matrix arithmetic in this class. Finally, we provide an effective implementation of the class of finitely representable QT matrices together with the related matrix arithmetic in the MATLAB toolbox cqt-toolbox.

In order to perform approximations of QT matrices with finitely representable matrices, we introduce the following norm

$$
\|A\|_{\mathcal{Q} \mathcal{T}}=\alpha\|a\|_{\mathcal{W}}+\|E\|_{2}, \quad \alpha=\frac{1+\sqrt{5}}{2} .
$$

This norm is different from the one used in [2,7,8, 13: it is slightly more general, and still makes the set $\mathcal{Q T}$ a Banach algebra. It can be shown that any value of $\alpha \geqslant \frac{1+\sqrt{5}}{2}$ would make this set a Banach algebra. Moreover, we will see that this choice allows a complete control on the approximation errors and enables us to perform, in a safe way, different computational operations like compression or matrix inversion.

The paper is organized as follows. In Section 2 we recall the definition and some theoretical results about QT-matrices, together with the norm $\|\cdot\|_{\mathcal{Q T}}$. We introduce the class of finitely representable QT matrices and provide a first description of the cqt-toolbox.

Section 3 deals with the definition and the analysis of the arithmetic operations in the algebra of finitely representable QT matrices. The first subsections deal with addition, multiplication, inversion and compression. Then, Section 3.5 describes the extension of the arithmetic (and of the toolbox) to the case of finite QT matrices. Section 4 provides some examples of applications, Section 5 draws the conclusions.

In the appendices, we provide some details on the Sieveking-Kung algorithm for triangular Toeplitz matrix inversion A.1, and on the main algorithms for computing the Wiener-Hopf factorization A.2. 


\section{The class of QT matrices}

We start by introducing the set of semi-infinite matrices that we are going to implement, recall its main properties and provide an effective (approximate) finite representation.

\subsection{The Wiener class and semi-infinite Toeplitz matrices}

We indicate with $\mathbb{T}:=\{z \in \mathbb{C}:|z|=1\}$ the complex unit circle, and with $\mathcal{W}$ the Wiener class formed by the functions $a(z)=\sum_{i=-\infty}^{+\infty} a_{i} z^{i}: \mathbb{T} \rightarrow \mathbb{C}$ such that $\sum_{i=-\infty}^{+\infty}\left|a_{i}\right|<+\infty$, that is functions expressed by a Laurent series with absolutely summable coefficients.

The set $\mathcal{W}$, endowed with the norm $\|a\|_{\mathcal{W}}:=\sum_{i \in \mathbb{Z}}\left|a_{i}\right|$, is a Banach algebra. By the Wiener theorem, [14, Section 1.4], a Laurent series in $\mathcal{W}$ is invertible if and only if $a(z) \neq 0$ on the unit circle. Under the latter condition, there exist functions $u(z)=\sum_{i=0}^{\infty} u_{i} z^{i}, l(z)=\sum_{i=0}^{\infty} l_{i} z^{i} \in \mathcal{W}$ with $u(z), l(z) \neq 0$ for $|z| \leqslant 1$ such that the factorization

$$
a(z)=u(z) z^{m} l\left(z^{-1}\right), \quad u(z)=\sum_{i=0}^{\infty} u_{i} z^{i}, \quad l(z)=\sum_{i=0}^{\infty} l_{i} z^{i}
$$

holds where $m$ is the winding number of $a(z)$. The above decomposition is known as Wiener-Hopf factorization. We refer the reader to the first chapter of the book 14 for more details.

We associate an element $a(z)=\sum_{i \in \mathbb{Z}} a_{i} z^{i}$ of the Wiener class with the semi-infinite Toeplitz matrix $T(a)=\left(t_{i, j}\right)$ such that $t_{i, j}=a_{j-i}$ for $i, j \in \mathbb{Z}^{+}$, or, in matrix form

$$
T(a)=\left[\begin{array}{cccc}
a_{0} & a_{1} & a_{2} & \ldots \\
a_{-1} & a_{0} & a_{1} & \ddots \\
a_{-2} & a_{-1} & a_{0} & \ddots \\
\vdots & \ddots & \ddots & \ddots
\end{array}\right] .
$$

Matrices $T(a)$ naturally define operators from $\ell^{2}$ into itself, where $\ell^{2}$ is the set of sequences $x=$ $\left(x_{i}\right)_{i \geqslant 1}$ such that $\|x\|_{2}=\left(\sum_{i=1}^{\infty}\left|x_{i}\right|^{2}\right)^{\frac{1}{2}}$ is finite. In particular, one can show that $\|T(a)\|_{2} \leqslant\|a\|_{\mathcal{W}}$, where $\|T(a)\|_{2}$ denotes the operator norm induced by the $\ell^{2}$-norm on the operator $T(a)$.

Given $a(z) \in \mathcal{W}$ we denote $a^{+}(z)=\sum_{i=1}^{\infty} a_{i} z^{i}, a^{-}(z)=\sum_{i=1}^{\infty} a_{-i} z^{i}$, so that we may write $a(z)=a^{-}\left(z^{-1}\right)+a_{0}+a^{+}(z)$. Moreover, given the power series $b(z)=\sum_{i=0}^{\infty} b_{i} z^{i} \in \mathcal{W}$, we denote $H(b)=\left(h_{i, j}\right)$ the semi-infinite Hankel matrix defined by $h_{i, j}=b_{i+j-1}$, for $i, j \in \mathbb{Z}^{+}$.

Despite $\mathcal{W}$ is closed under multiplication, the corresponding matrix class formed by semi-infinite Toeplitz matrices of the kind $T(a)$, for $a \in \mathcal{W}$, is not. However, it satisfies this property up to a compact correction [14] as stated by the following result.

Theorem 2.1. Let $a(z), b(z) \in \mathcal{W}$ and set $c(z)=a(z) b(z)$. Then

$$
T(a) T(b)=T(c)-H\left(a^{-}\right) H\left(b^{+}\right) .
$$

where $H\left(a^{-}\right)=\left(h_{i, j}^{-}\right)_{i, j \geqslant 1}, H\left(b^{+}\right)=\left(h_{i, j}^{+}\right)_{i, j \geqslant 1}$ with $h_{i, j}^{-}=a_{-(i+j+1)}$ and $h_{i, j}^{+}=b_{i+j+1}$. Moreover, the matrices $H\left(a^{-}\right)$and $H\left(b^{+}\right)$define compact operators on $\ell^{2}$ and are such that $\left\|H\left(a^{-}\right)\right\|_{2} \leqslant$ $\left\|a^{-}\right\|_{\mathcal{W}}$ and $\left\|H\left(b^{+}\right)\right\|_{2} \leqslant\left\|b^{+}\right\|_{\mathcal{W}}$. 
Assume that $a(z)=\sum_{-n_{-}}^{n_{+}} a_{i} z^{i}$ where $0 \leqslant n_{-}, n_{+}<\infty$. We recall that (see [17, [18]) for a continuous symbol $a(z)$ the matrix $T(a)$ is invertible if and only if $a(z) \neq 0$ for $|z|=1$ and the winding number of $a(z)$ is 0 . On the other hand, from [18, Theorem 1.14] the latter condition implies that there exist polynomials $u(z)=\sum_{i=0}^{n_{+}} u_{i} z^{i}$ and $l(z)=\sum_{i=0}^{n_{-}} l_{i} z^{i}$ having zeros of modulus less than 1 such that $a(z)=u(z) l\left(z^{-1}\right)$. Therefore we may conclude that if $T(a)$ is invertible then there exists the Wiener-Hopf factorization $a(z)=u(z) l\left(z^{-1}\right)$ so that $T(a)=T(u) T(l)^{T}$ and we may write

$$
T(a)^{-1}=\left(T(l)^{T}\right)^{-1} T(u)^{-1} .
$$

Observe that since $u(z)$ and $l(z)$ have zeros of modulus less than 1 , by Wiener's theorem, are invertible as power series. These arguments, together with Theorem 2.1, lead to the following result [17.

Theorem 2.2. If $a(z)=\sum_{-n_{-}}^{n_{+}} a_{i} z^{i}$, then $T(a)$ is invertible in $\ell^{2}$ if and only if there exists the Wiener-Hopf factorization $a(z)=u(z) l\left(z^{-1}\right)$, for $u(z)=\sum_{i=0}^{n_{+}} u_{i} z^{i}$ and $l(z)=\sum_{i=0}^{n_{-}} l_{i} z^{i}$ having zeros of modulus less than 1 . Moreover $u^{-1}(z), l^{-1}(z) \in \mathcal{W}$ so that

$$
\begin{aligned}
& a^{-1}(z)=l\left(z^{-1}\right)^{-1} u(z)^{-1}, \\
& T(a)^{-1}=T\left(l^{-1}\right)^{T} T\left(u^{-1}\right)=T\left(a^{-1}\right)+E, \quad E=H\left(l^{-1}\right) H\left(u^{-1}\right), \\
& \|E\|_{2} \leqslant\left\|l^{-1}\right\|_{\mathcal{W}}\left\|u^{-1}\right\|_{\mathcal{W}} .
\end{aligned}
$$

\subsection{Quasi-Toeplitz matrices}

We are ready to introduce the central notion of this paper.

Definition 2.3. We say that the semi-infinite matrix $A$ is Quasi-Toeplitz (QT) if it can be written in the form

$$
A=T(a)+E,
$$

where $a(z)=\sum_{i=-\infty}^{+\infty} a_{i} z^{i}$ is in the Wiener class, and $E=\left(e_{i, j}\right)$ defines a compact operator on $\ell^{2}$.

It is well known [17 that the class of $Q T$ matrices, equipped with the $\ell^{2}$ norm, is a Banach algebra. However, the $\ell^{2}$ norm can be difficult to compute numerically, so we prefer to introduce a slightly different norm which still preserves the Banach algebra property. Let $\alpha=(1+\sqrt{5}) / 2$ and set $\|A\|_{\mathcal{Q} \mathcal{T}}=\alpha\|a\|_{\mathcal{W}}+\|E\|_{2}$. Clearly, $\|A\|_{\mathcal{Q} \mathcal{T}}$ is a norm which makes complete the linear space of QT-matrices. Moreover, it is easy to verify that this norm is sub-multiplicative, that is, $\|A B\|_{\mathcal{Q T}} \leqslant\|A\|_{\mathcal{Q} \mathcal{T}}\|B\|_{\mathcal{Q} \mathcal{T}}$ for any pair of QT matrices $A, B$. This way, the linear space of QT matrices endowed with the norm $\|\cdot\|_{\mathcal{Q T}}$ forms a Banach algebra that we denote by $\mathcal{Q T}$. Observe also that $\|A\|_{2} \leqslant\|A\|_{\mathcal{Q} \mathcal{T}}$ for any QT matrix $A$.

The next lemma ensures that every QT matrix admits finitely representable approximations with arbitrary accuracy.

Lemma 2.4. Let $A=T(a)+E \in \mathcal{Q T}$ and $\epsilon>0$. Then, there exist non negative integers $n_{-}, n_{+}, n_{r}, n_{c}$ such that the matrix $\widehat{A}=T(\widehat{a})+\widehat{E}$, defined by

$$
\hat{a}(z)=\sum_{i=-n_{-}}^{n_{+}} a_{i} z^{i}, \quad \widehat{E}_{i j}=\left\{\begin{array}{ll}
E_{i j} & \text { if } 1 \leqslant i \leqslant n_{r} \quad \text { and } 1 \leqslant j \leqslant n_{c} \\
0 & \text { otherwise }
\end{array},\right.
$$

verifies $\|A-\widehat{A}\|_{\mathcal{Q T}} \leqslant\|A\|_{\mathcal{Q T}} \cdot \epsilon$. 
Proof. Since $A \in \mathcal{Q T}$ then $\|a\|_{\mathcal{W}}=\sum_{j \in \mathbb{Z}}\left|a_{j}\right|<\infty$. This means that there exist $n_{-}, n_{+}$such that

$$
\|a-\widehat{a}\|_{\mathcal{W}}=\sum_{j<-n_{-}}\left|a_{j}\right|+\sum_{j>-n_{+}}\left|a_{j}\right| \leqslant \epsilon\|A\|_{\mathcal{Q} \mathcal{T}} / \alpha .
$$

Since $E$ represents a compact operator, there exist $k \in \mathbb{N}, \sigma_{i} \in \mathbb{R}^{+}$and $u_{i}, v_{i} \in \mathbb{R}^{\mathbb{N}}$ with unit 2 -norm, $i=1, \ldots, k$, which verify $\left\|E-\sum_{i=1}^{k} \sigma_{i} u_{i} v_{i}^{T}\right\|_{2} \leqslant \frac{\epsilon}{2}\|E\|_{2}$. The condition $\left\|u_{i}\right\|_{2}=\left\|v_{i}\right\|_{2}=1$ implies that there exist two integers $n_{r}$ and $n_{c}$ such that the vectors

$$
\widetilde{u}_{i}(j)=\left\{\begin{array}{ll}
u_{i}(j) & \text { if } j>n_{r} \\
0 & \text { otherwise }
\end{array}, \quad \widetilde{v}_{i}(j)=\left\{\begin{array}{ll}
v_{i}(j) & \text { if } j>n_{c} \\
0 & \text { otherwise }
\end{array},\right.\right.
$$

have 2-norms bounded by $\frac{\epsilon\|A\|_{\mathcal{Q} \tau}}{4 k \max _{i} \sigma_{i}}$. Then, denoting by $\widehat{u}_{i}:=u_{i}-\widetilde{u}_{i}$ and $\widehat{v}_{i}:=v_{i}-\widetilde{v}_{i}$, and setting $\widehat{E}:=\sum_{i=1}^{k} \sigma_{i} \widehat{u}_{i} \widehat{v}_{i}^{T}$, we find that

$$
\left\|\widehat{u}_{i} \widehat{v}_{i}^{T}-u_{i} v_{i}^{T}\right\|_{2}=\left\|\widetilde{u}_{i} v_{i}^{T}+\widehat{u}_{i} \widetilde{v}_{i}^{T}\right\|_{2} \leqslant \frac{\epsilon\|A\|_{\mathcal{Q} \mathcal{T}}}{2 k \max _{i} \sigma_{i}} \Longrightarrow\left\|\widehat{E}-\sum_{i=1}^{k} \sigma_{i} u_{i} v_{i}^{T}\right\|_{2} \leqslant \frac{\epsilon}{2}\|A\|_{\mathcal{Q} \mathcal{T}} .
$$

To conclude, we have $\|E-\widehat{E}\|_{2} \leqslant\left\|E-\sum_{i=1}^{k} \sigma_{i} u_{i} v_{i}^{T}\right\|_{2}+\left\|\sum_{i=1}^{k} \sigma_{i} u_{i} v_{i}^{T}-\widehat{E}\right\|_{2} \leqslant \epsilon\|A\|_{\mathcal{Q} \mathcal{T}}$. Thus, from the latter inequality and from (3) we get $\|A-\widehat{A}\|_{\mathcal{Q} \mathcal{T}}=\alpha\|a-\hat{a}\|_{\mathcal{W}}+\|E-\widehat{E}\|_{2} \leqslant \epsilon\|A\|_{\mathcal{Q} \mathcal{T}}$.

This result makes it possible to draw an analogy between the representation of semi-infinite quasi Toeplitz matrices and floating point numbers. When representing a real number $a$ in floating point format $\mathrm{fl}(a)$ it is guaranteed that

$$
\mathrm{fl}(a)=a+\mathcal{E}, \quad|\mathcal{E}| \leqslant|a| \cdot \epsilon,
$$

where $\epsilon$ is the so-called unit roundoff.

We design a similar framework for QT-matrices. More precisely, analogously to the operator "fl(.)", we introduce a "truncation" operator $\mathcal{Q T}(\cdot)$ that works separately on the Toeplitz and on the compact correction, as described by Lemma 2.4. So, for a QT-matrix $A=T(a)+E_{a}$, we have

$$
\mathcal{Q T}(A)=T(\widehat{a})+\widehat{E}_{a}=A+\mathcal{E}, \quad\|\mathcal{E}\| \leqslant\|A\|_{\mathcal{Q} T} \cdot \epsilon,
$$

where $\epsilon$ is some prescribed tolerance set a priori (analogously to the unit roundoff), and $\mathcal{Q T}(A)$ is given by the sum of a banded Toeplitz matrix $T(\widehat{a})$ and a semi infinite matrix $\widehat{E}_{a}$, with finite support.

Matrices of the kind $\mathcal{Q T}(A)$ form the class of finitely representable Quasi Toeplitz matrices, where, unlike the case of floating point numbers, the lengths of the representations are not constant and may vary in order to guarantee a uniform bound to the relative error in norm.

The cqt-toolbox collects tools for operating with finitely representable Quasi Toeplitz matrices. The Toeplitz part is stored into two vectors containing the coefficients of the symbol with non positive and with non negative indices, respectively. The compact correction is represented in terms of two matrices $\widehat{U}_{a} \in \mathbb{R}^{n_{r} \times k}$ and $\widehat{V}_{a} \in \mathbb{R}^{n_{c} \times k}$ such that $\widehat{E}_{a}\left(1: n_{r}, 1: n_{c}\right)=\widehat{U}_{a} \widehat{V}_{a}^{T}$ coincides with the upper left corner of the correction.

In order to define a new finitely representable $\mathcal{Q T}$ matrix, one has to call the cqt constructor, in the following way: 
$1 \mid \gg \mathrm{A}=\operatorname{cqt}($ neg, pos, E);

In the above command, the vectors pos and neg contain the coefficients of the symbol $a(z)$ with non positive and non negative indices, respectively, and $\mathrm{E}$ is a finite section of the correction representing its non zero part. For example, to define a matrix $A=T(a)+E$ as follows

$$
A=\left[\begin{array}{cccccc}
1 & 2 & 1 & & & \\
-3 & 4 & 1 & 1 & & \\
& -1 & 2 & 1 & 1 & \\
& & \ddots & \ddots & \ddots & \ddots
\end{array}\right]=T(a)+E, \quad\left\{\begin{array}{l}
a(z)=-z^{-1}+2+z+z^{2} \\
E=\left[\begin{array}{ll}
-1 & 1 \\
-2 & 2
\end{array}\right]
\end{array}\right.
$$

one needs to type the following MATLAB commands:

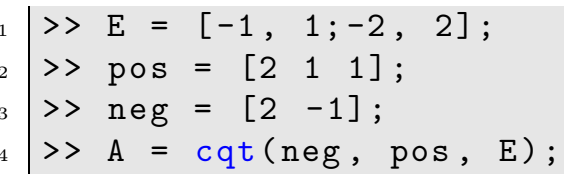

Notice that the constant coefficient is contains in both vectors, pos and neg. If the user supplies two different values, the toolbox returns an error. It is also possible to specify the correction in the factorized form $E=U V^{T}$.

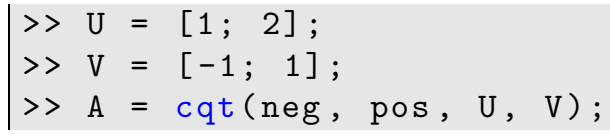

Removing the ; from the last expression will cause MATLAB to print a brief description of the infinite matrix.

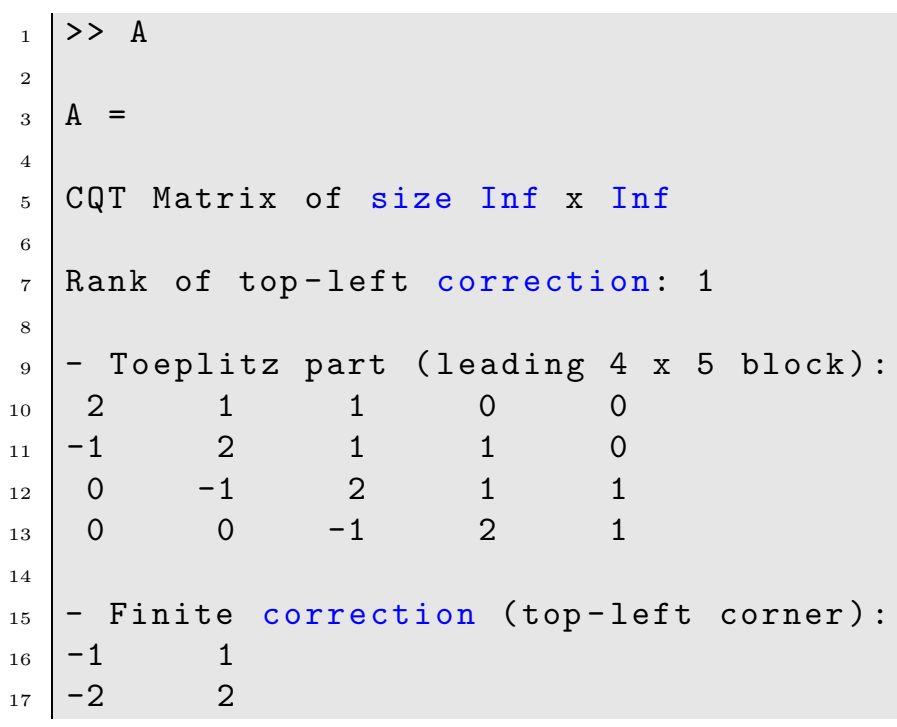

The different parts composing a $\mathcal{Q T}$ matrix $A$ can be fetched independently using the instructions symbol and correction. For the previous example we have: 


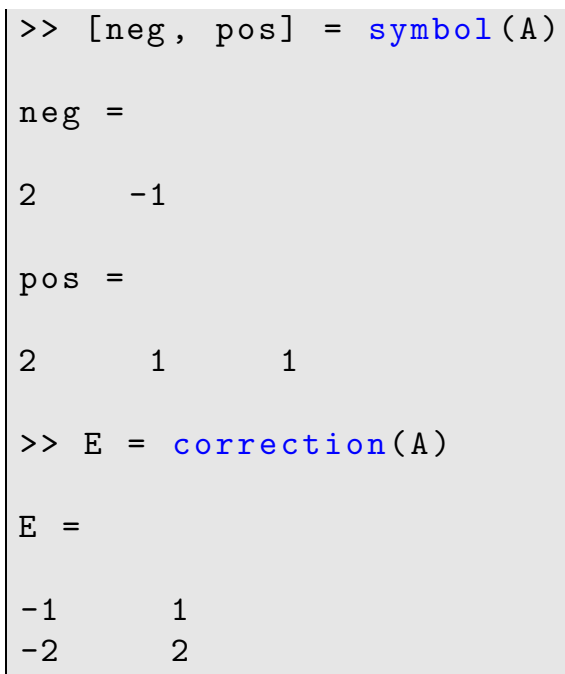

The command $[\mathrm{U}, \mathrm{V}]=\operatorname{correction}(\mathrm{A})$ allows to retrieve the correction in factorized form. The rank of the latter can be obtained with the command cqtrank.

\section{Arithmetic operations}

When performing floating point operations it is guaranteed that

$$
\mathrm{fl}(a \odot b)=a \odot b+\mathcal{E}, \quad|\mathcal{E}| \leqslant(a \odot b) \cdot \epsilon,
$$

where $\odot$ is any basilar arithmetic operation (sum, subtraction, multiplication, and division).

Extending the analogy, the matrix arithmetic in the set of finitely representable QT matrices is implemented in a way that the outcome of $A \odot B$, for any pair of finitely representable $A, B \in \mathcal{Q T}$ and $\odot \in\{+,-, *, /, \backslash\}$, is represented by $\mathcal{Q T}(A \odot B)$ such that

$$
A \odot B=\mathcal{Q T}(A \odot B)+\mathcal{E}, \quad\|\mathcal{E}\|_{\mathcal{Q T}} \leqslant \epsilon\|A \odot B\|_{\mathcal{Q} \mathcal{T}} .
$$

Notice that the outcome of an arithmetic operation between finitely representable QT matrices, might not be finitely representable, that is why we need to apply the $\mathcal{Q T}(\cdot)$ operator on it.

Another benefit of the $\mathcal{Q T}(\cdot)$ operator is that it optimizes the memory usage, since it minimizes the number of parameters required to store the data up to the required accuracy. The practical implementation of $\mathcal{Q T}(\cdot)$ is reported in Section 3.4 .

We now describe how the arithmetic operations are performed in the cqt-toolbox. These overloaded operators correspond to the built-in functions of MATLAB, i.e., they can be invoked with the usual operators $+,-, *, /, \backslash$. Since we represent only the non zero sections of infinite objects we rely on operations between matrices and vectors that might be of non compatible sizes, e.g., sum of vectors with different lengths. This has to be interpreted as filling the missing entries with zeros.

\subsection{Addition}

Given two finitely representable QT matrices $A=T(a)+E_{a}$ and $B=T(b)+E_{b}$, the matrix $C=A+B$ is defined by the symbol $c(z)=a(z)+b(z)$ and by the correction $E_{c}=E_{a}+E_{b}$. Hence, the symbol $c(z)$ is computed with two sums of vectors. The factorization $E_{c}=U_{c} V_{c}^{T}$ is given by 


$$
U_{c}=\left[U_{a}, U_{b}\right], \quad V_{c}=\left[V_{a}, V_{b}\right] .
$$

Then, applying the compression technique, where $U_{c}$ and $V_{c}$ are replaced by matrices $\widehat{U}_{c}$ and $\widehat{V}_{c}$, respectively, having a lower number of columns and such that $\left\|E_{c}-\widehat{U}_{c} \widehat{V}_{c}^{T}\right\|_{2}$ is sufficiently small, we get

$$
\mathcal{Q T}(A+B)=A+B+\mathcal{E}, \quad\|\mathcal{E}\|_{\mathcal{Q} \mathcal{T}} \leqslant \epsilon\|A+B\|_{\mathcal{Q} \mathcal{T}}
$$

The compression technique will be described in Section 3.4

We refer to $\mathcal{E}$ as the local error of the addition. Observe that if the operands $A$ and $B$ are affected themselves by an error $\mathcal{E}_{A}$ and $\mathcal{E}_{B}$, respectively, that is, the original QT-matrices $A$ and $B$ are represented by approximations $\widehat{A}$ and $\widehat{B}$, respectively such that

$$
\widehat{A}=A+\mathcal{E}_{A}, \quad \widehat{B}=B+\mathcal{E}_{B},
$$

then the computed matrix $\mathcal{Q} \mathcal{T}(\widehat{A}+\widehat{B})$ differs from $A+B$ by the total error given by

$$
\mathcal{Q} T(\widehat{A}+\widehat{B})-(A+B)=\mathcal{E}_{A}+\mathcal{E}_{B}+\mathcal{E}
$$

where $\mathcal{E}_{A}+\mathcal{E}_{B}$ is the inherent error caused by the approximated input, while $\mathcal{E}$ is the local error. Equation (8) says that the global error is the sum of the local error and the inherent error, and can be used to perform error analysis in the QT-matrix arithmetic.

\subsection{Multiplication}

In view of Theorem 2.1 we may write

$$
C=A B=T(c)-H\left(a^{-}\right) H\left(b^{+}\right)+T(a) E_{b}+E_{a} T(b)+E_{a} E_{b}=T(c)+E_{c},
$$

where $c(z)=a(z) b(z)$ and

$$
E_{c}=T(a) E_{b}+E_{a} T(b)+E_{a} E_{b}-H\left(a^{-}\right) H\left(b^{+}\right) .
$$

The symbol $c(z)$ is obtained by computing the convolution of the vectors representing the symbols $a(z)$ and $b(z)$, respectively.

For the correction part, denoting by $E_{a}=U_{a} V_{a}^{T}, E_{b}=U_{b} V_{b}^{T}, H\left(a^{-}\right)=M_{a} N_{a}^{T}, H\left(b^{+}\right)=M_{b} N_{b}^{T}$ the decompositions of the matrices involved, we may write $E_{c}=U_{c} V_{c}^{T}$ with

$$
U_{c}=\left[T(a) U_{b}, U_{a},-M_{a}\right], \quad V_{c}=\left[V_{b}, T(b)^{T} V_{a}+V_{b}\left(U_{b}^{T} V_{a}\right), N_{b}\left(M_{b}^{T} N_{a}\right)\right] .
$$

Notice that, the products $T(a) U_{b}$ and $T(b)^{T} V_{a}$ generate matrices with infinite rows and finite support. The computation of the non zero parts of the latter require only a finite section of $T(a)$ and $T(b)^{T}$, respectively. These operations are carried out efficiently relying on the fast Fourier transform (FFT).

The compressed outcome $\mathcal{Q} T(A B)$ satisfies the equation

$$
\mathcal{Q T}(A B)=A B+\mathcal{E}, \quad\|\mathcal{E}\|_{\mathcal{Q} \mathcal{T}} \leqslant \epsilon\|A B\|_{\mathcal{Q} \mathcal{T}},
$$


where $\mathcal{E}$ is the local error of the operation. If the operands $A$ and $B$ are affected by errors $\mathcal{E}_{A}$ and $\mathcal{E}_{B}$, respectively, such that (7) holds, then the global error in the computed product is given by

$$
\mathcal{Q T}(\widehat{A} \widehat{B})-A B=\mathcal{E}+A \mathcal{E}_{B}+B \mathcal{E}_{A}+\mathcal{E}_{A} \mathcal{E}_{B}
$$

where $A \mathcal{E}_{B}+B \mathcal{E}_{A}+\mathcal{E}_{A} \mathcal{E}_{B}$ is the inherent error caused by the approximated input, while $\mathcal{E}$ is the local error of the approximated multiplication. In a first order analysis we may replace the inherent error with $A \mathcal{E}_{B}+B \mathcal{E}_{A}$ neglecting the quadratic part $\mathcal{E}_{A} \mathcal{E}_{B}$.

\subsection{Inversion}

Let $A=T(a)+E_{a} \in \mathcal{Q T}$ be a finitely representable QT matrix such that the symbol

$$
a(z)=\sum_{i=-n_{a}^{-}}^{n_{a}^{+}} a_{i} z^{i}
$$

admits the Wiener-Hopf factorization in the form $a(z)=u(z) l\left(z^{-1}\right)$, so that $T(a)=T(u) T(l)^{T}$ is invertible and $T(a)^{-1}=\left(T(l)^{-1}\right)^{T} T(u)^{-1}$. Assume also that $E_{a}$ is given in the factored form $E_{a}=U_{a} V_{a}^{T}$ where $U_{a}$ and $V_{a}$ are matrices formed by $k$ columns and have null entries if the row index is greater than $m_{a}$.

Thus, we may write $A=T(a)\left(I+T(a)^{-1} E_{a}\right)$ so that, if $I+T(a)^{-1} E_{a}$ is invertible then also $A$ is invertible and

$$
A^{-1}=\left(I+T(a)^{-1} E_{a}\right)^{-1} T(a)^{-1}, \quad T(a)^{-1}=\left(T(l)^{-1}\right)^{T} T(u)^{-1} .
$$

Observe also that by Theorem 2.1 we may write $T(u)^{-1}=T\left(u^{-1}\right)$ and $T(l)^{-1}=T\left(l^{-1}\right)$.

Equation (10) provides a way to compute $A^{-1}$, represented in the QT form, which consists essentially in computing the coefficients of $u(z), l(z)$ and of their inverses, and then to invert a special QT matrix, that is, $I+T(a)^{-1} E_{a}=: I+E$.

Here we assume that the coefficients of the polynomials $u(z), l(z)$ and of the power series $u(z)^{-1}$ and $l(z)^{-1}$ are available. In the appendix, we provide more details on how to perform their computation. Once we have computed $u(z)^{-1}$ and $l(z)^{-1}$, by Theorem 2.1 we may write

$$
T(a)^{-1}=T(b)-H\left(l^{-1}\right) H\left(u^{-1}\right), \quad b(z)=l\left(z^{-1}\right)^{-1} u(z)^{-1},
$$

where the coefficients of $b(z)$ are computed by convolution of the coefficients of $u(z)^{-1}$ and of $l\left(z^{-1}\right)^{-1}$.

Concerning the inversion of $I+E$, where $E=T(a)^{-1} E_{a}$, we find that $E=T(a)^{-1} U_{a} V_{a}^{T}=$ : $U V^{T}$, for $U=T(a)^{-1} U_{a}, V=V_{a}$. Consider the $k \times k$ matrix $S_{k}=I_{k}+V^{T} U$ which has finite support since $V^{T} U=V_{a}^{T} T(a)^{-1} U_{a}$ and both $U_{a}$ and $V_{a}$ have a finite number of nonzero rows. If $S_{k}$ is invertible then it can be easily verified that $I-U S_{k}^{-1} V^{T}$ is the inverse of $I+U V^{T}$, that is, by the Shermann-Morrison formula,

$$
\left(I+U V^{T}\right)^{-1}=I-U S_{k}^{-1} V^{T}, \quad S_{k}=I_{k}+V^{T} U .
$$

Now, combining (10), (11), and (12) we may provide the following representation of the inverse:

$$
B:=A^{-1}=T(b)-H\left(l^{-1}\right) H\left(u^{-1}\right)-T\left(l^{-1}\right)^{T} T\left(u^{-1}\right) U_{a} S_{k}^{-1} V_{a}^{T} T\left(l^{-1}\right)^{T} T\left(u^{-1}\right) .
$$


Thus we may write $B$ in QT form as

$$
B:=T(b)+U_{b} V_{b}^{T}, \quad b(z)=l\left(z^{-1}\right)^{-1} u(z)^{-1},
$$

where

$$
U_{b}=\left[H\left(l^{-1}\right), T\left(l^{-1}\right)^{T} T\left(u^{-1}\right) U_{a} S_{k}^{-1}\right] \quad \text { and } \quad V_{b}=-\left[H\left(u^{-1}\right), T\left(u^{-1}\right)^{T} T\left(l^{-1}\right) V_{a}\right] .
$$

In order to analyze the approximation errors in computing $A^{-1}$ as a finitely representable QT matrix, we assume that the computed values of the Wiener-Hopf factors $u(z)$ and $l(z)$ are affected by some error and that also in the process of computing the inverse of a power series we introduce again some error. Therefore, we denote by $\widehat{u}(z)$ and $\widehat{l}(z)$ the computed values obtained in place of $u(z)$ and $l(z)$, respectively in the Wiener-Hopf factorization of $a(z)$ and set $e_{u l}(z)=a(z)-\widehat{u}(z) \widehat{l}\left(z^{-1}\right)$ for the residual error. Moreover, denote by $\delta_{u}(z)=u(z)-\widehat{u}(z), \delta_{l}(z)=l(z)-\widehat{l}(z)$ the absolute errors so that we may write the residual error as

$$
e_{u l}=l \delta_{u}+\widehat{u} \delta_{l} \doteq \widehat{l} \delta_{u}+\widehat{u} \delta_{l} .
$$

We indicate with $v(z)$ and $w(z)$ the power series reciprocal of $\widehat{u}(z)$ and $\widehat{l}(z)$, respectively, i.e., such that $\widehat{u}(z) v(z)=1$ and $\widehat{l}(z) w(z)=1$, while we denote with $\widehat{v}(z)$ and $\widehat{w}(z)$ the polynomials obtained by truncating $v(z)$ and $w(z)$ to a finite degree. Set $e_{u}(z)=\widehat{v}(z) \widehat{u}(z)-1, e_{l}(z)=\widehat{w}(z) \widehat{l}(z)-1$ for the corresponding residual errors. We approximate $a(z)^{-1}$ with the Laurent polynomial $\widehat{b}(z)=$ $\widehat{w}\left(z^{-1}\right) \widehat{v}(z)$ up to the error $e_{i n v}=a(z) \widehat{b}(z)-1$. Finally, we write $\doteq$ and $\dot{\leqslant}$ if the equality and the inequality, respectively, are valid up to quadratic terms in the errors $e_{u l}(z), e_{u}(z)$, and $e_{l}(z)$. This way, we may approximate the matrix $B=T(a)^{-1}=T\left(a^{-1}\right)-H\left(l^{-1}\right) H\left(u^{-1}\right)$ with the matrix $\widehat{B}=T(\widehat{b})-H(\widehat{w}) H(\widehat{v})$.

It is not complicated to relate $B-\widehat{B}$ to the errors $e_{i n v}(z), e_{u l}(z), e_{u}(z), e_{l}(z)$ as expressed in the following proposition where, for the sake of notational simplicity, we omit the variable $z$.

Proposition 3.1. The error $\mathcal{E}=T(a)^{-1}-\widehat{B}$, where $\widehat{B}=T(\widehat{b})-H(\widehat{w}) H(\widehat{v})$, is such that $\mathcal{E}=$ $-T\left(a^{-1} e_{i n v}\right)+E_{e}, E_{e}=H\left(l^{-1}-\widehat{w}\right) H\left(u^{-1}\right)+H(\widehat{w}) H\left(u^{-1}-\widehat{v}\right)$, and

$$
\left\|T\left(a^{-1} e_{i n v}\right)\right\|_{2} \leqslant\left\|a^{-1}\right\|_{\mathcal{W}}\left\|e_{i n v}\right\|_{\mathcal{W}}, \quad\left\|E_{e}\right\|_{2} \leqslant\left\|l^{-1}-\widehat{w}\right\|_{\mathcal{W}}\left\|u^{-1}\right\|_{\mathcal{W}}+\left\|u^{-1}-\widehat{v}\right\|_{\mathcal{W}}\|\widehat{w}\|_{\mathcal{W}} .
$$

Moreover, for the errors $e_{i n v}, e_{u}, e_{l}$ and $e_{u l}$ defined above it holds that

$$
e_{i n v} \doteq e_{u}+e_{l}+a^{-1} e_{u l} \doteq e_{u}+e_{l}+u^{-1} \delta_{u}+l^{-1} \delta_{l} .
$$

For the errors $l^{-1}-\widehat{w}$ and $u^{-1}-\widehat{v}$ it holds that

$$
\begin{aligned}
& l^{-1}-\widehat{w}=\left(l^{-1}-\widehat{l}^{-1}\right)+(w-\widehat{w})=-\widehat{l}^{-1}\left[l^{-1} \delta_{l}+e_{l}\right] \\
& u^{-1}-\widehat{v}=\left(u^{-1}-\widehat{u}^{-1}\right)+(v-\widehat{v})=-\widehat{u}^{-1}\left[u^{-1} \delta_{u}+e_{u}\right] .
\end{aligned}
$$

Proof. By linearity, we have $\mathcal{E}=T(a-\widehat{b})-H\left(l^{-1}\right) H\left(u^{-1}\right)+H(\widehat{w}) H(\widehat{v})=-T\left(a^{-1} e_{i n v}\right)+E_{e}$, where $E_{e}=H\left(l^{-1}-\widehat{w}\right)+H(\widehat{w}) H\left(u^{-1}-\widehat{v}\right)$, which, together with Theorem 2.1 proves the first part of the proposition. Observe that $a^{-1}=\left(e_{u l}+\widehat{u} \widehat{l}\right)^{-1} \doteq(\widehat{u} \widehat{l})^{-1}\left(1-(\widehat{u} \widehat{l})^{-1} e_{u l}\right)$ so that, since $\widehat{u}^{-1}=v$ and $\widehat{l}^{-1}=w$, we may write

$$
\widehat{b}-a^{-1}=\widehat{w} \widehat{v}-w v+(\widehat{u} \widehat{l})^{-2} e_{u l}=(\widehat{w}-w) \widehat{v}+w(\widehat{v}-v)(\widehat{u} \widehat{l})^{-2}+(\widehat{u} \widehat{l})^{-2} e_{u l} .
$$


Thus, since $\widehat{w}-w=\widehat{l}^{-1} e_{l} \doteq l^{-1} e_{l}$, and $\widehat{v}-v=\widehat{u}^{-1} e_{u} \doteq u^{-1} e_{u}$ we arrive at

$$
\widehat{b}-a^{-1} \doteq a\left(e_{l}+e_{u}+a e_{u l}\right),
$$

which proves (13). Equations (14) are an immediate consequence of the definitions of $e_{l}$ and $e_{u}$.

Proposition 3.1 enables one to provide an upper bound to $\left\|T(a)^{-1}-\widehat{B}\right\|$ in terms of $e_{l}, e_{u}, \delta_{l}$ and $\delta_{u}$ as shown in the following corollary.

Corollary 3.2. For the error $\mathcal{E}=T(a)^{-1}-\widehat{B}$ it holds

$$
\|\mathcal{E}\|_{\mathcal{Q} \mathcal{T}} \dot{\leqslant}\left(\alpha\left\|a^{-1}\right\|+\left\|u^{-1}\right\|_{\mathcal{W}}\left\|l^{-1}\right\|_{\mathcal{W}}\right)_{\mathcal{W}}\left(\left\|e_{u}\right\|_{\mathcal{W}}+\left\|e_{l}\right\|_{\mathcal{W}}+\left\|u^{-1}\right\|_{\mathcal{W}} \cdot\left\|\delta_{u}\right\|_{\mathcal{W}}+\left\|l^{-1}\right\|_{\mathcal{W}} \cdot\left\|\delta_{l}\right\|_{\mathcal{W}}\right) .
$$

A similar analysis can be performed for the errors in the computed inverse of $A=T(a)+E_{a}$. We omit the details.

We are ready to introduce a procedure to approximate the inverse of $T(a)$, which is reported in Algorithm 1. If the UL factorization cannot be computed, then the routine returns an error. The thresholds in the computation are adjusted to ensure that the final error is bounded by $\epsilon$. The symbol $b(z)$ of $T(a)^{-1}$ is returned, along with $\hat{v}(z)$ and $\hat{w}(z)$ such that $T(a)^{-1}=T(\widehat{b})+$ $H(\widehat{v}) H(\widehat{w})+\mathcal{E}$, where $\mathcal{E}=-T\left(a^{-1} e_{\text {inv }}\right)+E_{e}, E_{e}=H\left(\ell^{-1}-\widehat{w}\right) H\left(u^{-1}\right)+H(\widehat{w}) H\left(u^{-1}-\widehat{v}\right)$, and $\|\mathcal{E}\| \leqslant\left(\alpha\left\|a^{-1}\right\|_{\mathcal{W}}+\left\|u^{-1}\right\|_{\mathcal{W}}\left\|l^{-1}\right\|_{\mathcal{W}}\right) \epsilon$

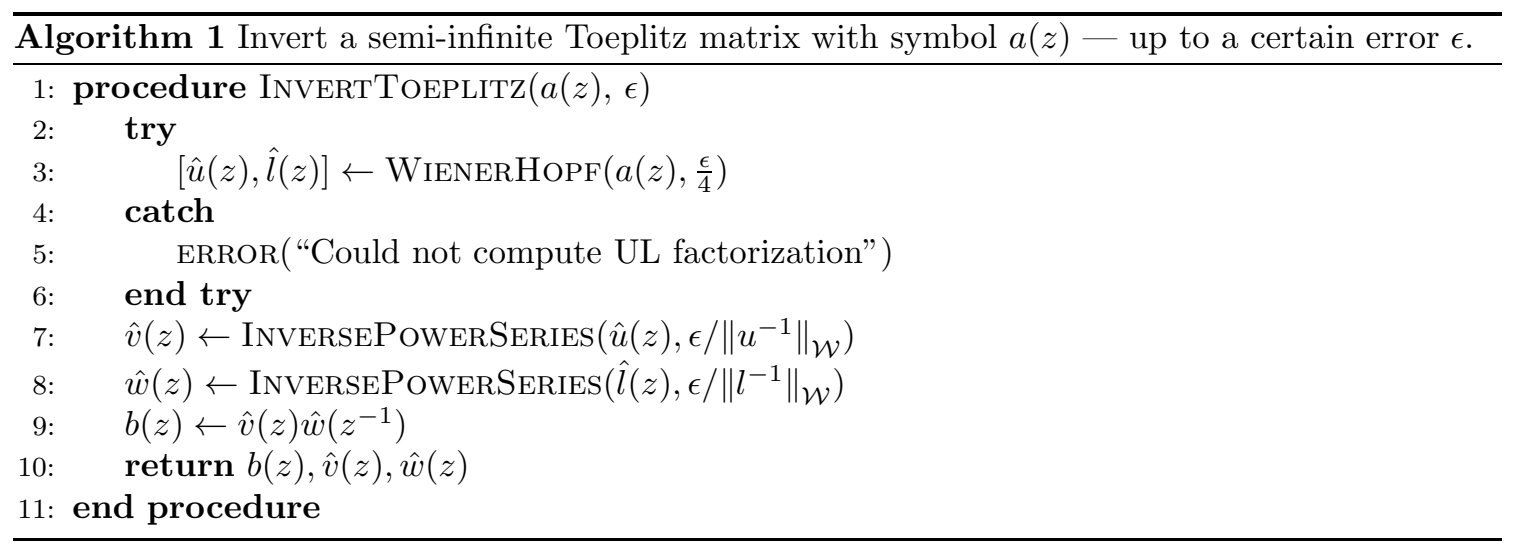

From Corollary 3.2 we find that $\|\mathcal{E}\|_{\mathcal{Q} \mathcal{T}} \leqslant\left(\alpha\left\|a^{-1}\right\|_{\mathcal{W}}+\left\|l^{-1}\right\|_{\mathcal{W}}\left\|u^{-1}\right\|_{\mathcal{W}}\right) \epsilon$. Thus, the correctness of the algorithm relies on Corollary 3.2 and on the existence of black boxes, which we will describe in the appendix, that implement the functions WIENERHopf $(\cdot)$ and $\operatorname{InVERSEPOWERSERIES}(\cdot)$. Relying on (10), a similar algorithm and analysis can be given for the computation of $\left(T(a)+E_{a}\right)^{-1}$.

\subsection{Truncation and compression}

We now describe in detail the implementation of the operator $\mathcal{Q T}$ on a finitely generated QT matrix. The truncation of a QT matrix $A=T(a)+E_{a}$ is performed as follows:

(i) Compute $\|A\|_{\mathcal{Q} \mathcal{T}}$. 
(ii) Obtain a truncated version $\hat{a}(z)$ of the symbol $a(z)$ by discarding the tails of the Laurent series. This has to be done ensuring that $\|a-\hat{a}\|_{\mathcal{W}} \leqslant\|A\|_{\mathcal{Q T}} \cdot \frac{\epsilon}{2 \alpha}$.

(iii) Compute a compressed version $\hat{E}_{a}$ of the correction using the SVD and dropping negligible rows and columns. Allow a truncation error bounded by $\|A\|_{\mathcal{Q T}} \cdot \frac{\epsilon}{2}$.

The above choices of thresholds provide an approximation $\hat{A}$ to $A$ such that $\|A-\hat{A}\|_{Q T} \leqslant$ $\|A\|_{Q T} \cdot \epsilon$. Notice that, the use of the QT-norm in the steps (ii) and (iii) enables to recognize unbalanced representations and to completely drop the negligible part.

When performing step (i), the only nontrivial step is to evaluate $\left\|E_{a}\right\|_{2}$. To this end, we compute an economy size SVD factorization of $E_{a}=U_{a} V_{a}^{T}$. This will also be useful in step (iii) to perform the low-rank compression.

In particular, we compute the $\mathrm{QR}$ factorizations $U_{a}=Q_{U} R_{U}, V_{a}=Q_{V} R_{V}$, so that, $U_{a} V_{a}^{T}=$ $Q_{U} R_{U} R_{V}^{T} Q_{V}^{T}$. Then, we compute the SVD of the matrix in the middle $R_{U} R_{V}^{T}=U_{R} \Sigma V_{R}^{T}$. We thus obtain an SVD of the form $E_{a}=U \Sigma V^{T}$, where $U=Q_{U} U_{R}$, and $V=Q_{V} V_{R}$. This is computed with $\mathcal{O}\left(n k^{2}\right)$ flops, where $n$ is the dominant dimension of the correction's support. The value of $\left\|E_{a}\right\|_{2}$ is obtained reading off the largest singular value, i.e., the $(1,1)$ entry of $\Sigma$.

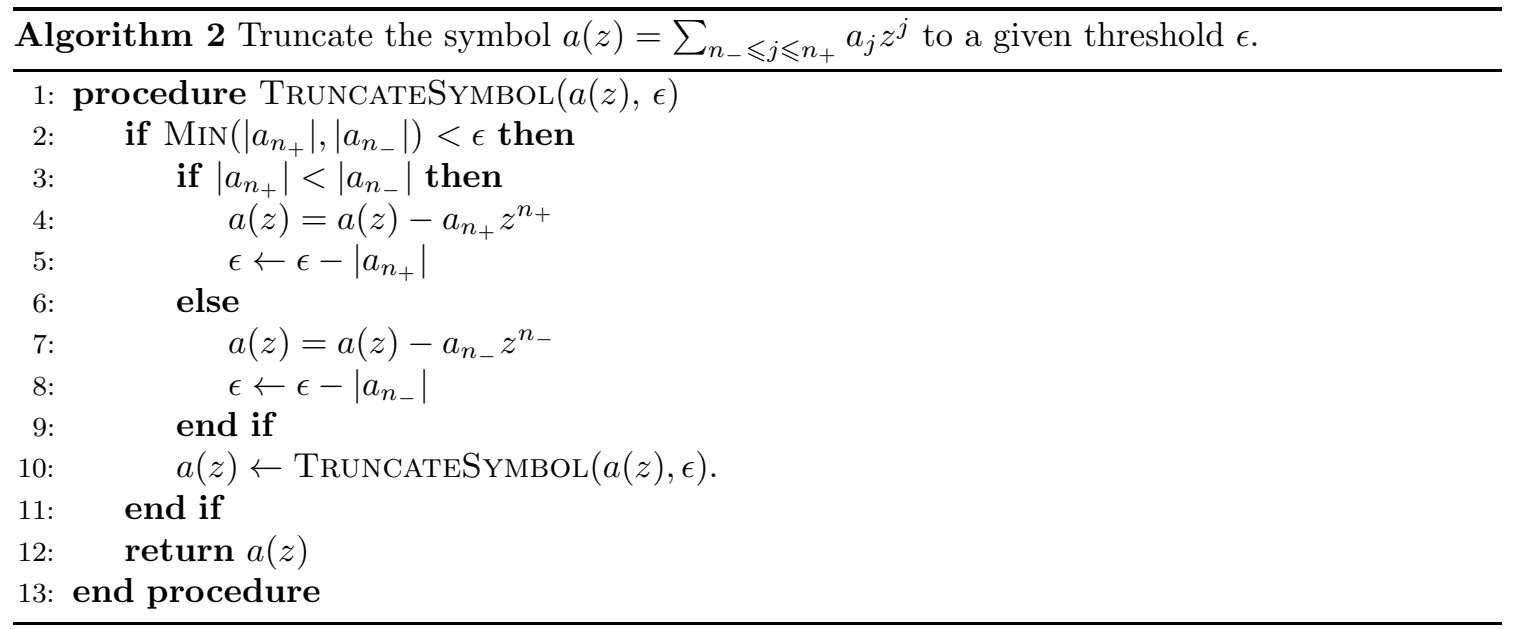

Concerning step (ii), we repeatedly discard the smallest of the extremal coefficients of $a(z)$, until the truncation errors do not exceed the specified threshold. In particular, we rely on Algorithm 2 using $\frac{\epsilon}{2 \alpha}\|A\|_{\mathcal{Q} \mathcal{T}}$ as second argument.

In step (iii), we first truncate the rank of $E_{a}$ by dropping singular values smaller than $\frac{\epsilon}{4} \cdot\|A\|_{\mathcal{Q} \mathcal{T}}$. To perform this step, we reuse the economy SVD computed at step (i). Then, we adopt a strategy similar to the one of Algorithm 2 to drop the last rows of $U$ and $V$. We set an initial threshold $\hat{\epsilon}=\frac{\epsilon}{4}\|A\|_{\mathcal{Q} \mathcal{T}}$, and we drop either the last row $U_{n}$ of $U$ or $V_{m}$ of $V$ if the norm of $U_{n} \Sigma$ (resp. $V_{m} \Sigma$ ) is smaller than the selected threshold. We then update $\hat{\epsilon}:=\hat{\epsilon}-\left\|U_{n} \Sigma\right\|$ (similarly for $V_{m} \Sigma$ ) and repeat the procedure until $\hat{\epsilon}>0$. This leads to a slightly pessimistic estimate, but ensures that the total truncation is within the desired bound. 


\subsubsection{Hankel compression}

When computing the multiplication of two Toeplitz matrices $T(a)$ and $T(b)$ it is necessary to store a low-rank approximation of $H\left(a^{-}\right) H\left(b^{+}\right)$(see Theorem 2.1). In fact, storing $H\left(a^{-}\right)$and $H\left(b^{+}\right)$ directly can be expensive whenever the Hankel matrices have large sizes e.g., when we multiply two QT-matrices having wide Toeplitz bandwidths. However, the numerical rank of the correction is often observed to be much lower than the dominant size of the correction's support. In such cases we exploit the Hankel structure to cheaply obtain a compressed representation $E_{c}=U_{c} V_{c}^{T}$. We call this task Hankel compression.

We propose two similar strategies for addressing Hankel compression. The first is to rely on a Lanczos-type method, in the form of the Golub-Kahan bidiagonalization procedure [33. This can be implemented by using matrix-vector products of the form $y=A x$ or $y=A^{T} x$, where $A=H\left(a^{-}\right) H\left(b^{+}\right)$is a product of two Hankel matrices. The product $y=A x$ can be computed in $\mathcal{O}(n \log n)$ time using the FFT. Since the Hankel matrices are symmetric, the multiplication by $A^{T}$ is obtained swapping the role of $a^{-}$and $b^{+}$.

This approach has an important advantage: the rank can be determined adaptively while the Lanczos process builds the basis, and assuming that Lanczos converges in $\mathcal{O}(k)$ steps, with $k$ being the numerical rank of $A$, then the complexity is $\mathcal{O}(k n \log n)$ flops: much lower than a full $\mathrm{QR}$ factorization or SVD.

A second (similar) strategy is to use random sampling techniques [20, which rely on the evaluation of the product $A U$, with $U$ being a matrix with normally distributed entries. If the columns of $A U$ span the range of $A$, then we extract an orthonormal basis of it, and we use it to cheaply compute the SVD of $A$ [20]. In the implementation the number of columns of $U$ is chosen adaptively, enlarging it until a sufficient accuracy is reached. The product $A U$ can be efficiently computed using the FFT, and it's possible to obtain BLAS3 speeds by re-blocking.

Both strategies are implemented in the package, and the user can select the Lanczos-type algorithm running cqtoption('compression', 'lanczos') or the one based on random sampling with the command: cqtoption('compression', 'random').

The performance of the two approaches is very similar. In Figure 1 the timings for the compression of the product of two $n \times n$ Hankel matrices are reported. The symbol has been chosen drawing from a uniform distribution enforcing an exponential decay as follows:

$$
a(z)=\sum_{j \in \mathbb{Z}^{+}} a_{j} z^{j}, \quad a_{j} \sim \lambda\left(0, e^{-\frac{j}{10}}\right),
$$

where $\lambda(a, b)$ is the uniform distribution on $[a, b]$. In the example reported in Figure1, the numerical rank (up to machine precision) of the product of the Hankel matrices generated according to (15) is 90. The break-even point with a full SVD is around 500 in this example, and this behavior is typical. Therefore, we use a dense singular value decomposition for small matrices $(n \leqslant 500)$, and we resort to Lanczos or random sampling (depending on user's preferences) otherwise.

In the right part of Figure 1 we report also the accuracies by taking the relative residual $\| U V^{T}-$ $H\left(a^{-}\right) H\left(b^{+}\right)\left\|_{2} /\right\| H\left(a^{-}\right) H\left(b^{+}\right) \|_{2}$. Since the norms are computed as dense matrices, we only test this up to $n=4096$. The truncation threshold in this example is set to $10^{-14}$.

\subsection{Finite quasi Toeplitz matrices}

The representation and the arithmetic, introduced so far, are here adapted for handling finite size matrices of the form Toeplitz plus correction. Clearly, all the matrices of finite size can be 


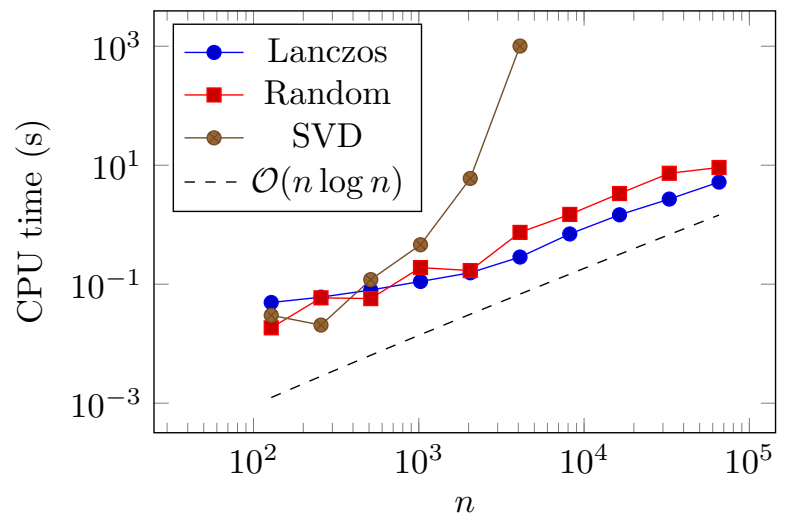

\section{Accuracy}

\begin{tabular}{ccc}
\hline Size & Lanczos & Random \\
\hline 128 & $1.85 \cdot 10^{-15}$ & $1.76 \cdot 10^{-15}$ \\
256 & $2.52 \cdot 10^{-15}$ & $5.78 \cdot 10^{-15}$ \\
512 & $1.48 \cdot 10^{-15}$ & $2.15 \cdot 10^{-15}$ \\
1,024 & $1.01 \cdot 10^{-15}$ & $1.69 \cdot 10^{-15}$ \\
2,048 & $7.84 \cdot 10^{-15}$ & $2.71 \cdot 10^{-15}$ \\
4,096 & $1.61 \cdot 10^{-15}$ & $1.03 \cdot 10^{-15}$
\end{tabular}

Figure 1: On the left, timings required to compress a product of two $n \times n$ Hankel matrices with decaying coefficients, for different values of $n$, and using different strategies. The tests with the dense singular value decomposition have been run only up to $n=4096$. The other methods have been tested up to $n=2^{16}$. On the right, the accuracies, up to size 4096, in the 2-norm achieved by setting the truncation threshold to $10^{-14}$.

represented in this way. This approach is convenient only if the corrections of the matrices involved in our computations are either sparse or low-rank. Typically, this happens when the input data of the computation are banded Toeplitz matrices.

In what follows, given a Laurent series $a(z)$ we indicate with $T_{n, m}(a)$ the $n \times m$ Toeplitz matrix obtained selecting the first $n$ rows and $m$ columns from $T(a)$. Given a power series $f(z)=\sum_{j \geqslant 0} f_{j} z^{j}$ we denote by $H_{n, m}(f)$ the $n \times m$ Hankel matrix whose non-zero anti-diagonal elements correspond to $f_{1}, f_{2}, \ldots, f_{\min \{n, m\}}$. Finally, given a Laurent polynomial $a(z)=\sum_{j=-n+1}^{m-1} a_{j} z^{j}$ we indicate with $\widetilde{a}(z)$ the shifted Laurent polynomial $z^{n-m} a(z)$.

In order to extend the approach, it is essential to look at the following variant of Theorem 2.1. for finite Toeplitz matrices 34 .

Theorem 3.3 (Widom). Let $a(z)=\sum_{-n+1}^{m-1} a_{j} z^{j}, b(z)=\sum_{-m+1}^{p-1} b_{j} z^{j}$ and $\operatorname{set} c(z)=a(z) b(z)$. Then

$T_{n, m}(a) T_{m, p}(b)=T(c)-H_{n, m}\left(a^{-}\right) H_{m, p}\left(b^{+}\right)-J_{n} H_{n, m}\left(\widetilde{a}^{+}\right) H_{m, p}\left(\widetilde{b}^{-}\right) J_{p}$
where $J_{n}=\left[\begin{array}{c}1 \\ . \cdot \\ 1\end{array}\right] \in \mathbb{R}^{n \times n}$ is the flip matrix.

An immediate consequence of Theorem 3.3 is the following extension of the Wiener-Hopf factorization for square Toeplitz matrices.

Corollary 3.4. Let $a(z)=\sum_{-n+1}^{n-1} a_{j} z^{j}$ and let $a(z)=u(z) l\left(z^{-1}\right)$ be its Wiener-Hopf factorization. Then

$$
T_{n, n}(a)=T_{n, n}(u) T_{n, n}(l)^{T}+J_{n} H_{n, n}(u) H_{n, n}(l) J_{n}
$$

The above results introduce an additional term with respect to their counterparts for semiinfinite matrices. In particular, if the lengths of the symbols involved is small, compared to the 
dimension of the matrices, then the support of the non-Toeplitz component is split in two parts located in the upper left corner and in the lower right corner, respectively. This suggests to consider two separate corrections.

Handling two separate corrections is convenient as long as they do not overlap. When this is the case, we represent finite quasi-Toeplitz matrices by storing two additional matrices, that represent the lower right correction in factorized form. More precisely, $A \in \mathbb{R}^{n \times m}$ is represented with two vectors, storing the symbol, and with the matrices $U_{a}, V_{a}, W_{a}, Z_{a}$ such that $U_{a} V_{a}^{T}$ and $J_{n} W_{a} Z_{a}^{T} J_{m}$ correspond to the corrections in the corners. As a practical example we report two possible options for defining the matrix

$$
A=\left[\begin{array}{ccccc}
1 & 3 & & & \\
-2 & \ddots & \ddots & & \\
& \ddots & \ddots & \ddots & \\
& & \ddots & \ddots & 3 \\
& & & -2 & 1
\end{array}\right]+\left[\begin{array}{ccccc}
1 & 1 & & & \\
1 & 1 & & & \\
& & & & \\
& & 1 & 2 & 3 \\
& & 2 & 4 & 6
\end{array}\right] \in \mathbb{R}^{12 \times 12} .
$$

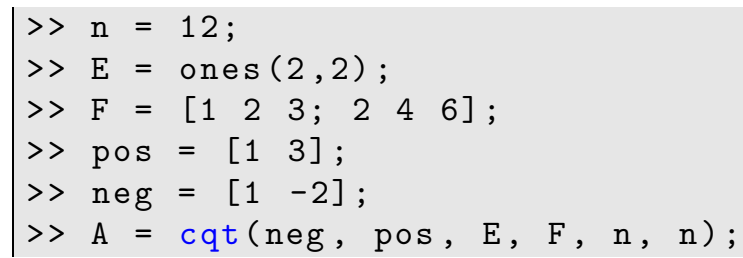

Once again, we also give the possibility to directly specify the corrections in the factorized form.

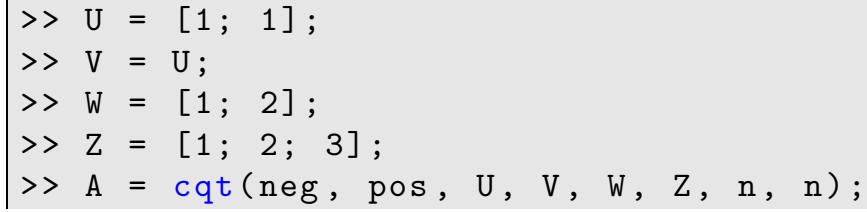

The arithmetic operations described for semi-infinite QT matrices can be analogously extended to this case. In the next section we describe in more detail how to handle the corrections when performing these operations.

In particular, when the corrections overlap, we switch to a single correction format, as in the semi-infinite case, where the support of the correction corresponds to the dimension of the matrix. In practice this is done by storing it as an upper-left correction, setting the lower right to the empty matrix. For this approach to be convenient, the rank of the correction needs to stay small compared to the size of the matrix. In fact, only the sparsity is lost, but the data-sparsity of the format is still exploitable.

\subsubsection{Handling the corrections in the computations}

Let $A=T(a)+U_{a} V_{a}^{T}+J_{n} W_{a} Z_{a}^{T} J_{n}$ and $B=T(b)+U_{b} V_{b}^{T}+J_{n} W_{b} Z_{b}^{T} J_{n}$. For simplicity, we assume that $A$ and $B$ are square, of dimension $n \times n$. Analogous statements hold in the rectangular case, which has been implemented in the toolbox. 


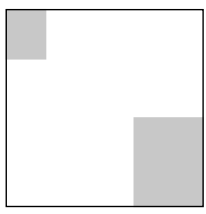

a)

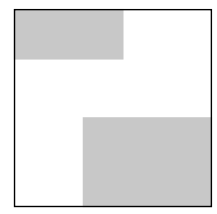

b)

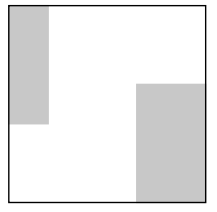

c)

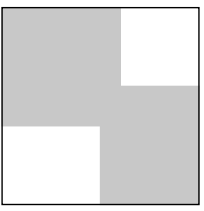

d)

Figure 2: Possible shapes of the corrections in the the representation of a finite QT-matrix. The corrections are disjoint in cases a) - c), but not in case d). In this last case it is convenient to store the correction entirely as a top correction.

As we already pointed out, we need to check that, while manipulating finite QT-matrices, the corrections do not overlap. More precisely, if the top-left correction of $A$ is of dimension $i_{u}^{(A)} \times j_{u}^{(A)}$ and the bottom one is $i_{l}^{(A)} \times j_{l}^{(A)}$, then we ask that at least one between $i_{u}^{(A)}+i_{l}^{(A)}$ and $j_{u}^{(A)}+j_{l}^{(A)}$ is smaller than $n$ (and analogous conditions for $B$ ). The possible configurations of the two corrections are reported in Figure 2] This property might not be preserved in the outcome of arithmetic operations.

Therefore, we need to understand how the supports of the corrections behave under sum and multiplications. Concerning the sum, the support of the correction in $A+B$ is contained in the union of the two supports in $A$ and $B$. The multiplication is slightly more involved. First, we check if the products $V_{a}^{T} J_{n} W_{b}$ and $Z_{a}^{T} J_{n} U_{b}$ are both zero, that is they do not "interact". This happens when $j_{u}^{(A)}+i_{l}^{(B)}$ and $j_{l}^{(A)}+i_{u}^{(B)}$ are both smaller than $n$. Second, we need to consider all the addends contributing to the correction; to this end, we note that the product of the top corrections has support $i_{u}^{(A)} \times j_{u}^{(B)}$ and the product of the bottom ones $i_{l}^{(A)} \times j_{l}^{(B)}$. Moreover, the multiplication of the Toeplitz part by the correction enlarges the support of the latter by the bandwidth of the former. Finally, the Hankel products have a support that depends on the length of the symbols. If the first condition is met and all these contributions satisfy the non-overlapping property for the sum, we keep the format with two separate corrections. Otherwise, we merge them into a single one. An analogous analysis is valid for the inversion, since it is implemented by a combination of sum and multiplications by means of the Woodbury formula.

\subsection{Other basic operations}

By exploiting the structure of the data, it is possible to devise efficient implementations of common operations, such as the computation of matrix functions, norms, and extraction of submatrices.

The functions reported in Table 1 have been implemented in the toolbox relying on the QT arithmetic. For a detailed description of the various functions, the user can invoke the help command. For instance, the matrix exponential is implemented using the Padé formula combined with a scaling and squaring strategy. An implementation based on the Taylor expansion is also available by calling expm (A, 'taylor').

In particular, the extraction of finite submatrices can be useful to inspect parts of infinite QT matrices, and also to obtain finite sections.

Remark 3.5. All the arithmetic operations, with the only exception of the inversion, can be performed in $\mathcal{O}(n \log n)$ time relying on the FFT. The current implementation of the Wiener-Hopf 


\begin{tabular}{cc} 
Function & Description \\
\hline $\mathrm{A}(\mathrm{I}, \mathrm{J})$ & Extract submatrices of a QT-matrix, for integer vectors $I$ and $J$. \\
$\mathrm{A}^{\wedge} \mathrm{p}$ & Integer power of a QT matrix. \\
$\mathrm{cr}$ & Cyclic reduction for quadratic matrix equations. \\
expm & Computation of the matrix exponential $e^{A}$. \\
funm & Computations of matrix functions by contour integration. \\
norm & Computation of the QT norm and, only in the finite case, of the $p$-norms for $p=1,2, \infty$. \\
sqrtm & Computation of the matrix square root (Denman-Beavers iteration). \\
ul & Compute the $U L$ factorization of any QT-matrix.
\end{tabular}

Table 1: List of implemented functions in cqt-toolbox.

factorization (see the Appendix), required for the inversion, needs $\mathcal{O}\left(b^{3}\right)$ where $b$ is the numerical bandwidth of the Toeplitz part - but this complexity can be lowered. For instance, one can obtain a sub-quadratic complexity combining the FFT-based polynomial inversion with a superfast Toeplitz solver for the computation of the Wiener-Hopf factorization. This is not currently implemented in the toolbox, and will be investigated in future work.

\section{Examples of applications}

In this section we show some applications and examples of computations with $\mathcal{Q T}$ matrices. Here we focus on the computation of matrix functions and solving matrix equations. Other examples related to matrix equations have already been shown in [2,8].

In all our experiments we have set the truncation tolerance to $\epsilon:=10^{-15}$. The algorithm used for compression is the Lanczos method. Accuracy and timings are analogous if the random sampling-based compression is used.

The tests have been performed on a laptop with an i7-7500U CPU running at $2.70 \mathrm{GHz}$ with 16GB of memory, using MATLAB R2017a.

\subsection{Exponential of Toeplitz matrices}

Recently there has been a growing interest in the computation of functions of Toeplitz matrices. For instance, in [29] the authors consider the problem of option pricing using the Merton model. This requires computing the exponential of a dense non-symmetric Toeplitz matrix. A fast method for this problem has been developed in 25 .

We refer to [29, Example 3] for the details on the model; the Toeplitz matrix obtained has symbol $a(z)$ with coefficients

$$
a_{j}=\left\{\begin{array}{ll}
\phi(0)+2 b-r-\lambda & j=0 \\
\phi\left(j \Delta_{\xi}\right)+b+j c & j= \pm 1 \\
\phi\left(j \Delta_{\xi}\right) & \text { otherwise }
\end{array} \quad, \quad b=\frac{\nu^{2}}{2 \Delta_{\xi}^{2}}, \quad c=\frac{2 r-2 \lambda \kappa-\nu^{2}}{4 \Delta_{\xi}}\right.
$$

where

$$
\phi(\eta):=\lambda \Delta_{\xi} \frac{e^{-(\eta-\mu)^{2} /\left(2 \sigma^{2}\right)}}{\sqrt{2 \pi} \sigma} .
$$




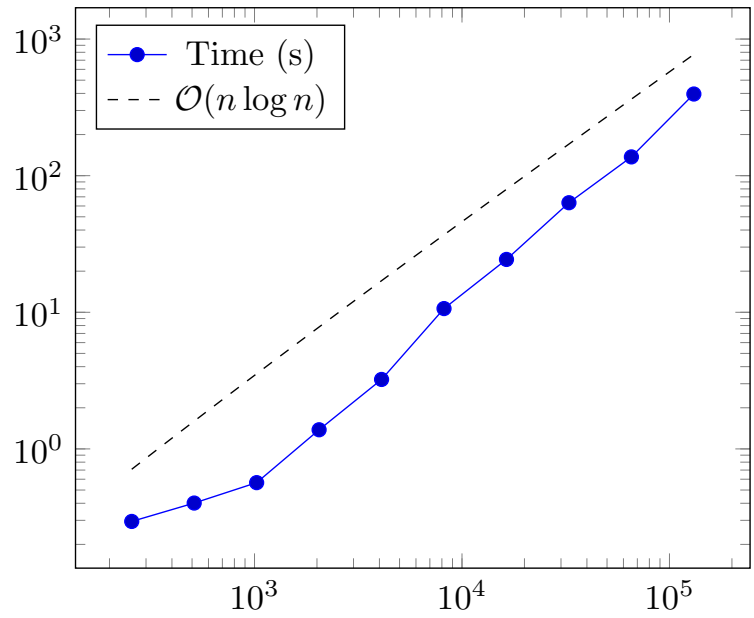

\begin{tabular}{ccc}
\hline Size & Time (s) & Corr. rank \\
\hline 256 & 0.29 & 42 \\
512 & 0.4 & 43 \\
1,024 & 0.57 & 43 \\
2,048 & 1.38 & 43 \\
4,096 & 3.22 & 43 \\
8,192 & 10.65 & 43 \\
16,384 & 24.37 & 44 \\
32,768 & 63.39 & 45 \\
65,536 & 137.2 & 46 \\
$1.31 \cdot 10^{5}$ & 396.05 & 49
\end{tabular}

Figure 3: Timings for the computation of the matrix exponential on the Merton model. The rank of the correction is reported in the last column of the table.

The values of the parameters are chosen as in [25]; for the Toeplitz matrix of size $n \times n$ we have:

$$
r=0.05, \quad \lambda=0.01, \quad \mu=-0.9, \quad \nu=0.25, \quad \sigma=0.45, \quad \kappa=e^{\frac{\mu+\sigma^{2}}{2}}-1, \quad \Delta_{\xi}=\frac{4}{n+1} .
$$

In Figure 3 we report the timings for the computation of the matrix exponential $e^{T_{n}}$ for different values of $n$. The CPU time is compared with the function $O(n \log n)$. The accuracy for the cases where $n \leqslant 4096$, where we could compare with expm, are reported in Figure 4 .

In particular, we report the relative error in the Frobenius norm $\|\operatorname{expm}(A)-E\|_{F} /\|\operatorname{expm}(A)\|_{F}$, where $E$ is the approximation of $e^{A}$ computed by the toolbox using the Taylor approximant. We compare it with the quantity $\|A\|_{F} \cdot \epsilon$, which is a lower bound for the condition number of the matrix exponential times the truncation threshold used in the computations (see [21]). From Figure 4 one can see that the errors are bounded by $\|A\|_{F} \cdot \epsilon$ and $10 \cdot\|A\|_{F} \cdot \epsilon$.

We have used a scaling and squaring scheme, combined with a Taylor approximant of order 12 , to compute the matrix exponential. In this case, where the bandwidth of the Toeplitz part is non-negligible, this approach is more efficient than a Padé approximant that requires an inversion (see Remark 3.5).

\subsection{Computing the square root of a semi-infinite matrix}

We show another application to the computation of the square root of an infinite QT-matrix $A$. We consider the infinite matrix $A=T(a)+E_{a}$, where

$$
a(z)=\frac{1}{4}\left(z^{-2}+z^{-1}+1+2 z+z^{2}\right),
$$

and $E_{a}$ is a rank 3 correction in the top-left corner of norm $\frac{1}{5}$ and support ranging from 32 to 1024 rows and columns. The square root can be computed using the Denman-Beavers iteration, which is implemented in the toolbox and accessible using $B=\operatorname{sqrtm}(A) ;$. We report the timings and the 


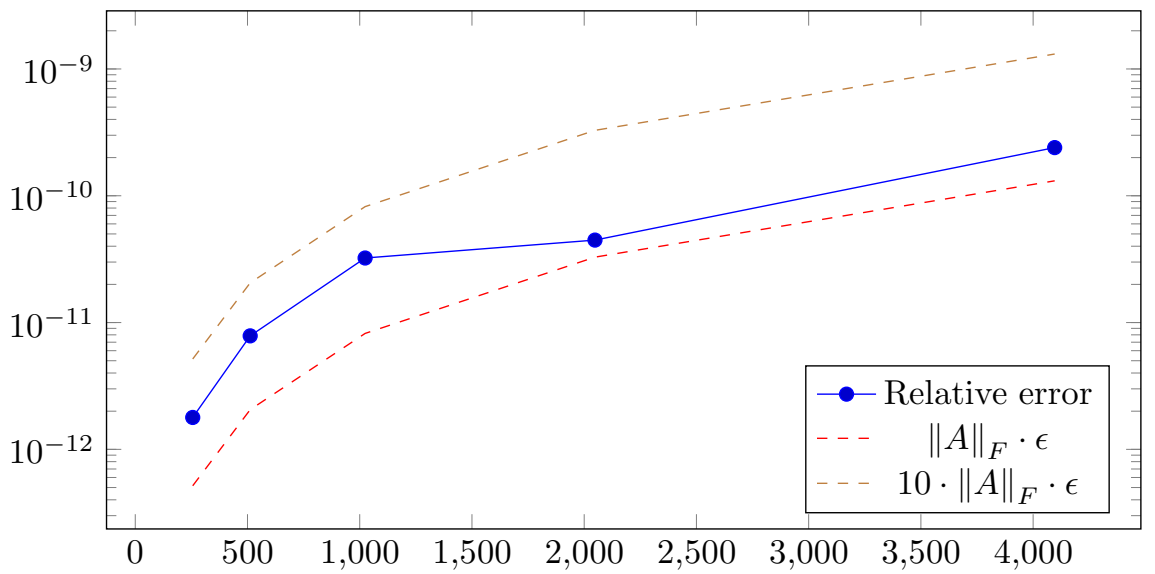

Figure 4: Relative error with the Frobenius norm of the computed matrix exponential, compared with a lower bound for the condition number of the matrix exponential multiplied by the truncation threshold used in the computation $\left(\epsilon:=10^{-15}\right)$.

\begin{tabular}{cccccc}
\hline Initial corr. support & Time $(\mathrm{s})$ & Residual & Rank & Corr. rows & Corr. cols \\
\hline 32 & 0.1 & $5.11 \cdot 10^{-14}$ & 34 & 268 & 285 \\
64 & 0.1 & $5.53 \cdot 10^{-14}$ & 38 & 296 & 316 \\
128 & 0.12 & $5.1 \cdot 10^{-14}$ & 39 & 357 & 379 \\
256 & 0.12 & $5.14 \cdot 10^{-14}$ & 39 & 476 & 507 \\
512 & 0.17 & $5.13 \cdot 10^{-14}$ & 39 & 726 & 744 \\
1,024 & 0.22 & $5.16 \cdot 10^{-14}$ & 39 & 1,226 & 1,271
\end{tabular}

Table 2: Timings and residuals for the computations of the square root of an infinite Toeplitz matrix with a square top-left correction of variable support. The final rank and correction support in the matrix $A^{\frac{1}{2}}$ are reported in the last 3 columns.

residual $\left\|B^{2}-A\right\|_{\mathcal{Q T}}$ of the computed approximations in Table 2, Moreover, the rank and support of the correction in $A^{\frac{1}{2}}$ are reported in the last three columns of the table. One can see that the rank stays bounded, and that the support does not increase much. The CPU time takes negligible values even for large support of the correction.

\subsection{Solving quadratic matrix equations}

Finally, we consider an example arising from the analysis of a random walk on the semi-infinite strip $\{0, \ldots, m\} \times \mathbb{N}$. We assume the random walk to be a Markov chain, and that movements are possible only to adjacent states, that is, from $(i, j)$ one can reach only $\left(i^{\prime}, j^{\prime}\right)$ with $\left|i-i^{\prime}\right|,\left|j-j^{\prime}\right| \leqslant 1$, with probabilities of moving up/down and left/right not depending on the current state. Then the 


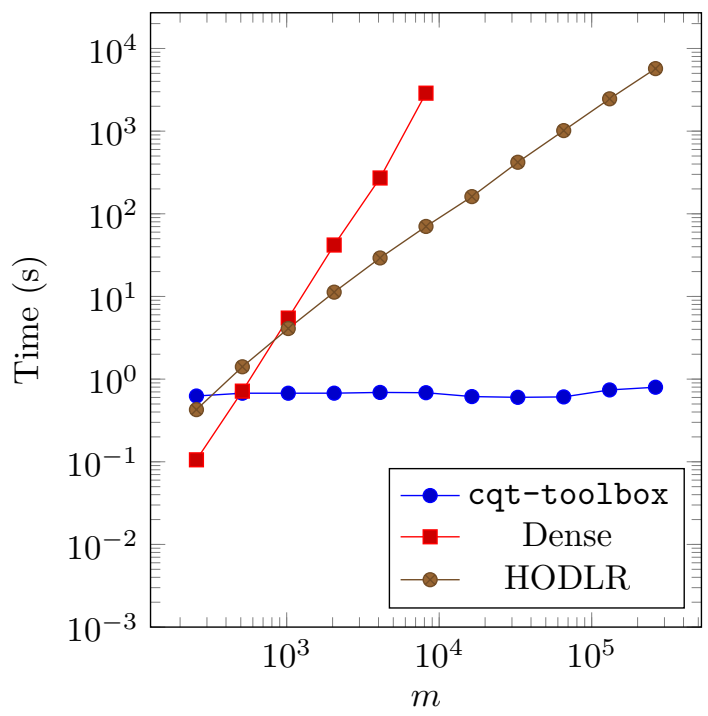

\begin{tabular}{ccccc}
\hline$m$ & $t_{\text {cqt }}$ & Corr. rank & $t_{\text {dense }}$ & $t_{\text {HODLR }}$ \\
\hline 256 & 0.62 & 19 & 0.11 & 0.43 \\
512 & 0.68 & 19 & 0.72 & 1.41 \\
1,024 & 0.68 & 19 & 5.48 & 4.09 \\
2,048 & 0.68 & 19 & 41.91 & 11.27 \\
4,096 & 0.69 & 19 & 271.2 & 29.23 \\
8,192 & 0.68 & 19 & $2,879.7$ & 70.32 \\
16,384 & 0.61 & 19 & & 161.41 \\
32,768 & 0.6 & 19 & & 420.29 \\
65,536 & 0.61 & 19 & & $1,015.5$ \\
$1.31 \cdot 10^{5}$ & 0.74 & 19 & & 2,460 \\
$2.62 \cdot 10^{5}$ & 0.8 & 19 & & $5,707.4$
\end{tabular}

Figure 5: On the left, timings for the solution of the quadratic equation $A_{-1}+A_{0} G+A_{1} G^{2}=G$ arising from the random walk on an infinite strip. On the right the timings and the ranks of the final correction are reported in the table.

transition matrix $P$ is an infinite Quasi-Toeplitz-Block-Quasi-Toeplitz matrix of the form

$$
P=\left[\begin{array}{cccc}
\hat{A}_{0} & A_{1} & & \\
A_{-1} & A_{0} & A_{1} & \\
& \ddots & \ddots & \ddots
\end{array}\right],
$$

and the problem of computing the invariant vector $\pi$ requires to solve the $m \times m$ quadratic matrix equation $A_{-1}+A_{0} G+A_{1} G^{2}=G\left[12\right.$. The matrices $A_{i}$ are non negative tridiagonal Toeplitz matrices with corrections to the elements in position $(1,1)$ and $(m, m)$, and satisfy $\left(A_{-1}+A_{0}+\right.$ $\left.A_{1}\right) e=e$, where $e$ is the vector of all ones.

The solution $G$ can be computed, for instance, using Cyclic reduction (see the Appendix for the details) - a matrix iteration involving matrix products and inversions. We consider an example where the transition probabilities are chosen in a way that gives the following symbols:

$$
a_{-1}(z)=\frac{1}{4}\left(2 z^{-1}+2+2 z\right), \quad a_{0}(z)=\frac{1}{10}\left(z^{-1}+2 z\right), \quad a_{1}(z)=\frac{1}{6}\left(3 z^{-1}+6+2 z\right),
$$

properly rescaled in order to make $A_{-1}+A_{0}+A_{1}$ a row-stochastic matrix. The top and bottom correction are chosen to ensure stochasticity on the first and last row.

We compare the performances of a dense iteration (without exploiting any structure) - with the same one implemented using cqt-toolbox, and also with a fast $\mathcal{O}\left(m \log ^{2} m\right)$ method which exploits the tridiagonal structure relying on the arithmetic of hierarchical matrices (HODLR) [9]10. In Figure 5, one can see that the timings of the dense solver are lower for small dimensions - but 
the ones using the toolbox do not suffer from the increase in the dimension. The dense solver was tested only up to dimension $m=4096$.

The implementation relying on cqt-toolbox is faster already for dimension 512, and has the remarkable property that the time does not depend on the dimension. This is to be expected, since the computations are all done on the symbol (which is dimension independent), and on the corrections, which only affect top and bottom parts of the matrices.

The residual of the quadratic matrix equation $\left\|A_{-1}+A_{0} G+A_{1} G^{2}\right\|$ is bounded in the $\mathcal{Q} T$ norm by approximately $7 \cdot 10^{-12}$ in all the tests, independently of the dimension, when the cqt-toolbox solver is used.

\section{Conclusions}

We have analyzed the class of Quasi Toeplitz matrices, introduced a suitable norm and a way to approximate any QT matrix by means of a finitely representable matrix within a given relative error bound. Within this class, we have introduced and analyzed, in all the computational aspects, a matrix arithmetic. We have provided an implementation of QT matrices and of their matrix arithmetic in the form of a Matlab toolbox. The software cqt-toolbox, available at https://github.com/numpi/cqt-toolbox, has been tested with both semi-infinite QT matrices and with finite matrices represented as the sum of a Toeplitz matrix and a correction. This software has shown to be very efficient in computing matrix functions and solving matrix equations encountered in different applications.

\section{A Appendix}

Here we report the main algorithms that we have implemented to perform inversion of QT matrices, namely, the Sieveking-Kung algorithm [3] for inverting triangular Toepliz matrices (or power series), and an algorithm based on Cyclic Reduction [12 to compute the Wiener-Hopf factorization of a symbol $a(z)$. We also provide a general view of the available algorithms for the Wiener-Hopf factorization [4, [5], [16, with an outline of their relevant computational properties. Choosing the more convenient algorithm for this factorization depends on several aspects like the degree of $a(z)$, and the location of its zeros, and this is an issue to be better understood.

\section{A.1 The Sieveking-Kung algorithm}

We shortly recall the Sieveking-Kung algorithm for computing the first $k+1$ coefficients $v_{0}, \ldots, v_{k}$ of $v(z)=\sum_{i=0}^{\infty} v_{i} z^{i}$ such that $v(z) u(z)=1$, or equivalently, the first $k$ entries in the first row of $T(u)^{-1}$. For more details we refer the reader to the book [3].

For notational simplicity, denote $V_{q}$ the $q \times q$ leading submatrix of $T(u)$. Consider $V_{2 q}$ and partition it into 4 square blocks of size $q$ :

$$
V_{2 q}=\left[\begin{array}{cc}
V_{q} & S_{q} \\
0 & V_{q}
\end{array}\right]
$$

so that

$$
V_{2 q}^{-1}=\left[\begin{array}{cc}
V_{q}^{-1} & -V_{q}^{-1} S_{q} V_{q}^{-1} \\
0 & V_{q}^{-1}
\end{array}\right]
$$


Since the inverse of an upper triangular Toeplitz matrix is still upper triangular and Toeplitz, it is sufficient to compute the first row of $V_{2 q}^{-1}$. The first half clearly coincides with the first row of $V_{q}^{-1}$, the second half is given by $-e_{1}^{T} V_{q}^{-1} S_{q} V_{q}^{-1}$, where $e_{i}$ is the vector with the $i$-th component equal to 1 and with the remaining components equal to zero.

Thus the algorithm works this way: For a given (small) $q$ compute the first $q$ components by solving the system $V_{q}^{T} x=e_{1}$. Then, by subsequent doubling steps, compute $2 q, 4 q, 8 q, \ldots$, components until some stop condition is satisfied. Observe that, denoting $v_{q}(z)$ the polynomial obtained at step $q$, the residual error $r_{q}(z)=a(z) v_{q}(z)-1$ can be easily computed so that the stop condition $\left\|r_{q}\right\|_{\mathcal{W}} \leqslant \epsilon\|a\|_{\mathcal{W}}$ can be immediately implemented. Concerning the convergence speed, it must be pointed out that the equation $r_{2 q}(z)=r_{q}(z)^{2}$ holds true (see [3]), implying that the convergence to zero of the norm of the residual error is quadratic.

This approach has a low computational cost since the products Toeplitz matrix by vector can be implemented by means of FFT for an overall cost of the Sieveking-Kung algorithm of $O(n \log n)$ arithmetic operations.

This algorithm, here described in matrix form, can be equivalently rephrased in terms of polynomials and power series.

\section{A.2 The Wiener-Hopf factorization}

We recall and synthesize the available algorithms for computing the coefficients of the polynomials $u(z)$ and $l(z)$ such that $a(z)=u(z) l\left(z^{-1}\right)$ is the Wiener-Hopf factorization of $a(z)$. Denote $\xi_{i}$ the zeros of $a(z)$ ordered so that $\left|\xi_{i}\right| \leqslant\left|\xi_{i+1}\right|$. This way, $\left|\xi_{n_{+}}\right|<1<\left|\xi_{1+n_{+}}\right|$, moreover, $u\left(\xi_{i}\right)=0$ for $i=1, \ldots, n_{+}$while $l\left(\xi_{i}^{-1}\right)=0$ for $i=n_{+}+1, \ldots, n_{+}+n_{-}$.

A first approach is based on reducing the problem to solving a quadratic matrix equation. Let $p \geqslant \max \left(n_{-}, n_{+}\right)$, reblock the matrices in the equation $T(a)=T(u) T\left(l^{-1}\right)$ into $p \times p$ blocks and obtain

$$
\left[\begin{array}{cccc}
A_{0} & A_{1} & & \\
A_{-1} & A_{0} & A_{1} & \\
& \ddots & \ddots & \ddots
\end{array}\right]=\left[\begin{array}{llll}
U_{0} & U_{1} & & \\
& U_{0} & U_{1} & \\
& & \ddots & \ddots
\end{array}\right]\left[\begin{array}{cccc}
L_{0} & & & \\
L_{1} & L_{0} & & \\
& L_{1} & L_{0} & \\
& & \ddots & \ddots
\end{array}\right]
$$

where, by using the MATLAB notation,

$$
\begin{aligned}
& A_{0}=\operatorname{toeplitz}\left(\left[a_{0}, \ldots, a_{-p+1}\right],\left[a_{0}, \ldots, a_{p-1}\right]\right), \\
& A_{1}=\operatorname{toeplitz}\left(\left[a_{p}, a_{p-1}, \ldots, a_{1}\right],\left[a_{p}, 0, \ldots, 0\right]\right), \\
& A_{-1}=\operatorname{toeplitz}\left(\left[a_{-p}, 0, \ldots, 0\right],\left[a_{-p}, \ldots, a_{-1}\right]\right),
\end{aligned}
$$

and $a_{i}=0$ if $i$ is out of range.

Set $W=U_{0} L_{0}, R=-U_{1} U_{0}^{-1}, G=-L_{0}^{-1} L_{1}$ and get the factorization

$$
\left[\begin{array}{cccc}
A_{0} & A_{1} & & \\
A_{-1} & A_{0} & A_{1} & \\
& \ddots & \ddots & \ddots
\end{array}\right]=\left[\begin{array}{cccc}
I & -R & & \\
& I & -R & \\
& & \ddots & \ddots
\end{array}\right]\left[\begin{array}{lll}
W & & \\
& W & \\
& & \ddots
\end{array}\right]\left[\begin{array}{cccc}
I & & & \\
-G & I & & \\
& -G & I & \\
& & \ddots & \ddots
\end{array}\right] .
$$

Multiplying the above equation to the right by the block column vector with entries $I, G, G^{2}, G^{3}, \ldots$ or multiplying to the left by the block row vector with entries $I, R, R^{2}, R^{3}, \ldots$ one finds that the 
matrices $R$ and $G$ are solutions of the equations

$$
A_{1} G^{2}+A_{0} G+A_{-1}=0, \quad R^{2} A_{-1}+R A_{0}+A_{1}=0
$$

and have eigenvalues $\xi_{1}, \ldots, \xi_{n_{+}}$and $\xi_{n_{+}+1}^{-1}, \ldots, \xi_{n_{+}+n_{-}}^{-1}$, respectively, so that they have spectral radius less than 1 . For more details in this regard we refer the reader to 6 .

Observe that, since

$$
G=-\left[\begin{array}{cccc}
l_{0} & & & \\
l_{1} & l_{0} & & \\
\vdots & \ddots & \ddots & \\
l_{p-1} & \ldots & l_{1} & l_{0}
\end{array}\right]^{-1}\left[\begin{array}{ccc}
l_{p} & \ldots & l_{1} \\
& \ddots & \vdots \\
& & l_{p}
\end{array}\right]
$$

then $G e_{p-n_{-}+1}=-l_{n_{-}} L_{0}^{-1} e_{1}$, while $e_{1}^{T} G=-l_{0}^{-1}\left(l_{p}, \ldots, l_{1}\right)$. That is, the first row of $G$ provides the coefficients of the factor $l(z)$ normalized so that $l_{0}=-1$. Similarly, one finds that $R e_{1}=$ $-u_{0}^{-1}\left(u_{p}, u_{p-1}, \ldots, u_{1}\right)^{T}$, and $e_{p-n_{+}+1}^{T} R=-u_{n_{+}} e_{1}^{T} U_{0}^{-1}$. That is, the first column of $R$ provides the coefficients of the factor $u(x)$ normalized so that $u_{0}=-1$. In order to determine the normalizing constant $w$ such that $a(z)=u(z) w l\left(z^{-1}\right)$, it is sufficient to impose the condition $u_{n_{+}} w l_{0}=a_{n_{+}}$so that we can choose $w=-a_{n_{+}} / u_{n_{+}}$.

This argument provides the following algorithm to compute the coefficients of $l(x)$ and of $u(x)$ such that $a(z)=u(z) w l(1 / z)$, where $u_{0}=l_{0}=-1$ :

1. Assemble the matrices $A_{-1}, A_{0}, A_{1}$.

2. Determine $R$ and $G$ that solve (16) using cyclic reduction.

3. Compute $\hat{u}=R e_{1}$, set $u=\left(-1, \hat{u}_{p}, \ldots, \hat{u}_{1}\right)$ and $\hat{v}=e_{1}^{T} G$, set $l=\left(-1, \hat{v}_{p}, \ldots, \hat{v}_{1}\right)$.

4. Set $w=-a_{n_{+}} / u_{n_{+}}$

Observe that the above algorithm can be easily modified in order to compute, for a given $q$, the first $q$ coefficients of the triangular Toeplitz matrices $\mathcal{U}^{-1}$ ad $\mathcal{L}^{-1}$ such that

$$
\mathcal{A}^{-1}=\frac{1}{w} \mathcal{L}^{-1} \mathcal{U}^{-1}
$$

In fact, the first $p$ coefficients, are given by

$$
\begin{aligned}
& L_{0}^{-1} e_{1}=-\frac{1}{l_{n_{-}}} G e_{p-n_{-}+1}, \\
& e_{1}^{T} U_{0}^{-1}=-\frac{1}{u_{n_{+}}} e_{p-n_{+}+1}^{T} R .
\end{aligned}
$$

While the remaining coefficients can be computed by means of the Sieveking-Kung algorithm described in the previous section.

The method described in this section requires the computation of the solutions $G$ and $R$ of equation (16). One of the most effective methods to perform this computation is the Cyclic Reduction (CR) algorithm. We refer the reader to 12 for a review of this method, and to 11 for the analysis of the specific structural and computational properties of the matrices generated in this way. Here 
we provide a short outline of the algorithm, applied to the equations (16) which we rewrite in the form $A G^{2}+B G+C=0$ and $R^{2} C+R B+A=0$, respectively. The algorithm CR computes the following matrix sequences

$$
\begin{aligned}
& B^{(k+1)}=B^{(k)}-A^{(k)} S^{(k)} C^{(k)}-C^{(k)} S^{(k)} B^{(k)}, \quad S^{(k)}=\left(B^{(k)}\right)^{-1}, \\
& A^{(k+1)}=-A^{(k)} S^{(k)} A^{(k)}, \quad C^{(k+1)}=-C^{(k)} S^{(k)} C^{(k)}, \\
& \widehat{B}^{(k+1)}=\widehat{B}^{(k)}-C^{(k)}\left(B^{(k)}\right)^{-1} A^{(k)}, \quad \widetilde{B}^{(k+1)}=\widetilde{B}^{(k)}-A^{(k)}\left(B^{(k)}\right)^{-1} C^{(k)} .
\end{aligned}
$$

It is proved that under mild conditions the sequences can be computed with no breakdown and that $\left.\lim _{k}-A\left(\widehat{B}^{k)}\right)^{-1}=R, \lim _{k}-\widetilde{(} B^{(k)}\right)^{-1} C=G$. More precisely, the following relations hold

$$
\begin{aligned}
& \left.G=-\left(\widetilde{B}^{(k)}\right)^{-1} C-\widetilde{(} B^{(k)}\right)^{-1} A^{(k)} G^{2^{k}} \\
& \left.R=-A\left(\widehat{B}^{(k)}\right)^{-1}-R^{2^{k}} C^{(k)} \widehat{(} B^{(k)}\right)^{-1}
\end{aligned}
$$

and it can be proved that $\left\|\left(\widehat{B}^{(k)}\right)^{-1}\right\|$ and $\left\|\left(\widetilde{B}^{(k)}\right)^{-1}\right\|$ are uniformly bounded by a constant and that $A^{(k)}, C^{(k)}$ converge double exponentially to zero. Since the spectral radii of $R$ and of $G$ are less than 1, this fact implies that convergence is quadratic. Moreover, the approximation errors given by the matrices $\left(\widetilde{B}^{(k)}\right)^{-1} A^{(k)} G^{2^{k}}$ and $R^{2^{k}} C^{(k)}\left(\widehat{B}^{(k)}\right)^{-1}$ is explicitely known in a first order error analysis. In fact the matrices $\left(\widetilde{B}^{(k)}\right)^{-1},\left(\widehat{B}^{(k)}\right)^{-1}, A^{(k)}$ and $C^{(k)}$ are explicitely computed by the algorithm and $G$ is approximated. This fact allows us to implement effectively the Wiener-Hopf computation required in the inversion procedure described in Algorithm 1 of Section 3.3.

The cost of Cyclic Reduction is $O\left(p^{3}\right)$ arithmetic operations per step. In [11] it is shown that all the above matrix sequences are formed by matrices having displacement rank bounded by small constants. This fact enables one to implement the above equation with a linear cost, up to logarithmic factors, by means of FFT.

\section{A.2.1 A different approach}

Another approach to compute the factor $l$ and $u$ relies on the following property [4].

Theorem A.1. Let $a(z)^{-1}=h(z)=\sum_{i=-\infty}^{\infty} h_{i} z^{i}$. Define the Toeplitz matrix of size $q \geqslant \max (m, n)$ $T_{q}=\left(h_{j-i}\right)$. Then, $T_{q}$ is invertible and its last row and column define the coefficient vectors of $l(z)$ and $u(z)$, respectively up to a normalization constant.

Proof. The relation $a(z)^{-1}=l^{-1}\left(z^{-1}\right) u^{-1}(z)$ can be rewritten in matrix form as

$$
\left(h_{j-i}\right)=T\left(u^{-1}\right) T\left(l^{-1}\right)^{T} .
$$

Multiply to the right by the infinite vector obtained by completing $\left(l_{0}, \ldots, l_{q-1}\right)$ with zeros. Since the product of $T\left(l^{-1}\right)^{T}$ with the latter is a vector with all null components except the first one, equal to 1 , considering $q$ components of the result yields

$$
T_{q}(h)\left(l_{0}, \ldots, l_{q-1}\right)^{T}=\left(T\left(u^{-1}\right)\right) e_{1}=u_{0}^{-1} e_{q}
$$

whence we deduce that $\left(l_{0}, \ldots, l_{q-1}\right)^{T}=T_{q}(h)^{-1} u_{0}^{-1} e_{q}$. Similarly we do for the last row.

This property is at the basis of the following computations 
1. Set $q=\max (m, n)$ compute $h_{i}$ for $i=-q, q$ such that $h(z) a(z)=1$ by means of evaluation/interpolation.

2. Form $T_{q}(h)=\left(h_{j-i}\right)_{i, j=1, q}$ and compute last row and last column of $T_{q}(h)^{-1}$.

This algorithm may require a large number of interpolation points when $a(z)$ has some zero of modulus close to 1 , in the process of evaluation/interpolation.

\section{A.2.2 Yet another approach}

The same property provides a third algorithm for computing $l(z)$ and $u(z)$ which relies on a different computation of $h_{i}, i=-q, \ldots, q$. The idea is described below

Consider the equation

$$
a(z) h(z)=1 .
$$

Multiply it by $a(-z)$ and, since $a(-z) a(z)=a_{1}\left(z^{2}\right)$, for a polynomial $a_{1}(z)$, get

$$
a_{1}\left(z^{2}\right) h(z)=a(-z) .
$$

Repeating the procedure $k$ times yields

$$
a_{k}\left(z^{2^{k}}\right) h(z)=a_{k-1}\left(-z^{2^{k-1}}\right) \cdots a_{1}\left(-z^{2}\right) a(-z) .
$$

If $a(z)$ has roots of modulus different from 1 , then $a_{k}(z)$ quickly converges to either a constant or a scalar multiple of $z$, since its zeros are the $2^{k}$ powers of the zeros of $a(z)$. In this case, $h(z)$ can be computed by means of a product of polynomials with the same degree (independent of the iterations).

\section{A.2.3 Newton's iteration}

Newton's iteration can be applied to the nonlinear system $a(z)=u(z) l\left(z^{-1}\right)$ where the unknowns are the coefficients of the polynomials $u(z)$ and $l(z)$. The Jacobian matrix has a particular structure given in terms of displacement rank which can be exploited to implement Newton's iteration at a low cost. Details in this regard are given in the papers [16] and [15].

\section{References}

[1] N. Bean and G. Latouche. Approximations to quasi-birth-and-death processes with infinite blocks. Adv. in Appl. Probab., 42(4):1102-1125, 2010.

[2] D. Bini, S. Massei, B. Meini, and L. Robol. On quadratic matrix equations with infinite size coefficients encountered in QBD stochastic processes. Numer. Linear Algebra Appl., in press.

[3] D. Bini and V. Y. Pan. Polynomial and matrix computations. Vol. 1. Progress in Theoretical Computer Science. Birkhäuser Boston, Inc., Boston, MA, 1994. Fundamental algorithms.

[4] D. A. Bini and A. Böttcher. Polynomial factorization through Toeplitz matrix computations. Linear Algebra Appl., 366:25-37, 2003. 
[5] D. A. Bini, G. Fiorentino, L. Gemignani, and B. Meini. Effective fast algorithms for polynomial spectral factorization. Numer. Algorithms, 34(2-4):217-227, 2003.

[6] D. A. Bini, L. Gemignani, and B. Meini. Computations with infinite Toeplitz matrices and polynomials. Linear Algebra Appl., 343/344:21-61, 2002.

[7] D. A. Bini, S. Massei, and B. Meini. On functions of quasi Toeplitz matrices. Sb. Math., 208(11):56-74, 2017.

[8] D. A. Bini, S. Massei, and B. Meini. Semi-infinite quasi-Toeplitz matrices with applications to QBD stochastic processes. Math. Comp., 2018.

[9] D. A. Bini, S. Massei, and L. Robol. Efficient cyclic reduction for quasi-birth-death problems with rank structured blocks. Appl. Numer. Math., 116:37-46, 2017.

[10] D. A. Bini, S. Massei, and L. Robol. On the decay of the off-diagonal singular values in cyclic reduction. Linear Algebra Appl., 519:27-53, 2017.

[11] D. A. Bini and B. Meini. Effective methods for solving banded Toeplitz systems. SIAM J. Matrix Anal. Appl., 20(3):700-719, 1999.

[12] D. A. Bini and B. Meini. The cyclic reduction algorithm: from Poisson equation to stochastic processes and beyond. Numer. Algorithms, 51(1):23-60, 2009.

[13] D. A. Bini and B. Meini. On the exponential of semi-infinite quasi-Toeplitz matrices. arXiv preprint arXiv:1611.06380, 2016.

[14] A. Böttcher and S. M. Grudsky. Spectral properties of banded Toeplitz matrices. Siam, 2005.

[15] A. Böttcher and M. Halwass. A Newton method for canonical Wiener-Hopf and spectral factorization of matrix polynomials. Electron. J. Linear Algebra, 26:873-897, 2013.

[16] A. Böttcher and M. Halwass. Wiener-Hopf and spectral factorization of real polynomials by Newton's method. Linear Algebra Appl., 438(12):4760-4805, 2013.

[17] A. Böttcher and B. Silbermann. Introduction to large truncated Toeplitz matrices. Springer Science \& Business Media, 2012.

[18] I. C. Gohberg. On an application of the theory of normed rings to singular integral equations. Uspehi Matem. Nauk (N.S.), 7(2(48)):149-156, 1952.

[19] J. Gutiérrez-Gutiérrez, P. M. Crespo, and A. Böttcher. Functions of the banded Hermitian block Toeplitz matrices in signal processing. Linear Algebra Appl., 422(2-3):788-807, 2007.

[20] N. Halko, P.-G. Martinsson, and J. A. Tropp. Finding structure with randomness: Probabilistic algorithms for constructing approximate matrix decompositions. SIAM Rev., 53(2):217-288, 2011.

[21] N. J. Higham. Functions of matrices: theory and computation. SIAM, 2008.

[22] J. R. Jackson. Networks of waiting lines. Oper. Res., 5(4):518-521, 1957. 
[23] S. Kapodistria and Z. Palmowski. Matrix geometric approach for random walks: Stability condition and equilibrium distribution. Stoch. Models, 33(4):572-597, 2017.

[24] M. Kobayashi and M. Miyazawa. Revisiting the tail asymptotics of the double QBD process: refinement and complete solutions for the coordinate and diagonal directions. In Matrixanalytic methods in stochastic models, volume 27 of Springer Proc. Math. Stat., pages 145-185. Springer, New York, 2013.

[25] D. Kressner and R. Luce. Fast Computation of the Matrix Exponential for a Toeplitz Matrix. SIAM J. Matrix Anal. Appl., 39(1):23-47, 2018.

[26] G. Latouche, G. T. Nguyen, and P. G. Taylor. Queues with boundary assistance: the effects of truncation. Queueing Syst., 69(2):175-197, 2011.

[27] G. Latouche and V. Ramaswami. Introduction to Matrix Analytic Methods in Stochastic Modeling. ASA-SIAM Series on Statistics and Applied Probability. SIAM, Philadelphia PA, 1999.

[28] G. Latouche and P. Taylor. Truncation and augmentation of level-independent QBD processes. Stochastic Process. Appl., 99(1):53-80, 2002.

[29] S. T. Lee, H.-K. Pang, and H.-W. Sun. Shift-invert Arnoldi approximation to the Toeplitz matrix exponential. SIAM J. Sci. Comput., 32(2):774-792, 2010.

[30] M. Lindner. Infinite matrices and their finite sections. Frontiers in Mathematics. Birkhäuser Verlag, Basel, 2006. An introduction to the limit operator method.

[31] M. Miyazawa. Light tail asymptotics in multidimensional reflecting processes for queueing networks. Top, 19(2):233-299, 2011.

[32] M. F. Neuts. Matrix-geometric solutions in stochastic models: an algorithmic approach. Courier Dover Publications, 1981.

[33] C. C. Paige. Bidiagonalization of matrices and solutions of the linear equations. SIAM J. Numer. Anal., 11:197-209, 1974.

[34] H. Widom. Asymptotic behavior of block Toeplitz matrices and determinants. II. Advances in Math., 21(1):1-29, 1976. 